\title{
Incipient Voltage Sensor Fault Isolation for Rectifier in Railway Electrical Traction Systems
}

\author{
Kangkang Zhang, Bin Jiang, Senior Member, IEEE, Xing-Gang Yan and Zehui Mao
}

\begin{abstract}
This paper proposes a dc voltage incipient sensor fault isolation method for single-phase three-level rectifier devices in high-speed railway electrical traction systems. Different incipient fault modes characterizing locations and incipient fault types are parameterized nonlinearly by unknown fault parameters. A new incipient fault isolation method is developed by combining sliding mode technique with nonlinear parametrization adaptive estimation technique. A bank of particular adaptive sliding mode estimators is proposed, which facilitates to derive new isolation residuals and adaptive threshold intervals. The isolability is studied, and the isolable sufficient condition is derived using new functions. For the practical electrical traction system in CRH2 (China Railway High-Speed 2), simulation and experiment based on TDCS-FIB (a software) are presented to verify the effectiveness and feasibility of the proposed method.
\end{abstract}

Index Terms-Rectifiers dc voltage sensors, fault isolation, sliding mode and adaptive techniques.

\section{INTRODUCTION}

C HINA high-speed railways are fed by $2 \times 25 \mathrm{KV} / 50 \mathrm{~Hz}$ single phase ac current sources [1]. The ac/dc/ac electrical traction system in $\mathrm{CRH} 2$ includes a single-phase threelevel ac-dc rectifier, a three-phase dc-ac inverter and four driving motors where the rectifier is different from the twolevel rectifier discussed in [2]. Such a power electronic system may experience static electricity corrosion, high humidity and high temperature, electrical loading and mechanical vibration. For example, the electrical traction drive for an urban tram may experience $10^{6}-10^{8}$ power cycles in its lifetime, with temperature swings up to $80^{\circ} \mathrm{C}$ [3]. In addition, aging components in sensors, such as electrolyte loss in electrolytic capacitors which are most fragile [4], may result in incipient faults and further develop into serious failures. Therefore, early incipient sensor fault diagnosis is essential and significant.

Manuscript received Month $\mathrm{xx}, 2 \mathrm{xxx}$; revised Month $\mathrm{xx}, \mathrm{xxxx}$; accepted Month $\mathrm{x}, \mathrm{xxxx}$. This work is supported in part by the National Natural Science Foundation of China (Grant 61490703, 61573180), the Fundamental Research Funds for the Central Universities (NO. NE2014202), the Priority Academic Program Development of Jiangsu Higher Education Institutions, the Research Innovation Program for College Graduates of Jiangsu Province (KYLX_160374). (Corresponding author: Bin Jiang)

Kangkang Zhang, Bin Jiang and Zehui Mao are with the College of Automation Engineering, Nanjing University of Aeronautics and Astronautics, Nanjing210016, China (e-mail: kangzhang359@163.com; binjiang@nuaa.edu.cn; zehuimao@nuaa.edu.cn).

Xing-gang Yan is with School of Engineering and Digital Arts, University of Kent, Canterbury, Kent CT2 7NT, United Kingdom (e-mail: x.yan@kent.ac.uk).
During the past decades, model-based fault diagnosis has been widely studied and applied such as [5] and [6]. The sliding mode observer based FDI (fault detection and isolation) has been extensively studied [7], [8] and [9]. In [7] and [8], the "equivalent output injection" concept is used to explicitly reconstruct fault signals to detect and isolate sensor faults and actuator faults. Using a sliding mode observer, an actuator FDI scheme is developed by generating residuals instead of reconstructing fault signals in [9]. All the above sliding mode observer based methods require that the fault signals are bounded by known functions. In addition, the challenging problem of residuals' convergence for mismatched fault modes is not considered in [9]. Adaptive is a significant technique to solve these problems, especially to deal with uncertainties such as [10] and [11], and adaptive observer based FDI has been studied in [12], [13], [14], etc.. However, residuals and adaptive thresholds generated in these papers are conservative. Sliding mode technique in combination with adaptive technique is a pertinent solution to improve robustness of FDI against modeling uncertainties and disturbances. In [15] and [16], the adaptive and sliding mode techniques are used to estimate fault signals. However, residual based fault isolation by combining adaptive approach together with sliding mode technique is rarely few. In practical rectifiers, there always exist switching noises and grid-side voltage and current harmonics. However, sliding mode techniques have good robustness and are completely insensitive to the so-called matched uncertainty, and can also be used to deal with both structural and unmatched uncertainty (see e.g. [17]). They are usually used to control and monitor electrical equipments such as rectifiers, inverters and induction motors [18]. Therefore, the application of sliding mode techniques for rectifier incipient voltage sensor fault isolation offers good potential.

In this paper, a multi incipient sensor faults isolation method is developed for single-phase three-level rectifier dc voltage sensors in high-speed railway electrical traction systems. The voltage incipient sensor faults are modeled by differential equations with unknown inputs parameterized by fault parameters in a nonlinear way. To isolate different fault modes, a bank of particular designed sliding mode and adaptive estimators is proposed. In the FIEs (fault isolation estimators), the projection adaptive laws are proposed based on Min-Max method proposed in [19] to estimate the fault parameters. In addition, all the estimation errors generated by FIEs are ensured to enter into the sliding surface in finite time and maintain on it thereafter, no matter whether the matched or mismatched fault modes occur. New residuals are defined, 
and adaptive threshold intervals are designed based on the interval observer theory (see e.g., [20]). The incipient sensor fault mode isolable condition characterizing which fault mode can be isolated, is derived by new defined functions. The effectiveness and feasibility of the proposed dc voltage incipient sensor fault isolation method is tested using the TDCS-FIB program provided by Central South University fault injection team.

Notations: For a real matrix or a vector $M, M>0(M \geq 0)$ means that its entries are positive (nonnegative). The symbol $\operatorname{diag}(v)$ denotes a diagonal matrix with the diagonal elements formed by the elements of the vector $v$. The identity matrix with dimension $n$ is denoted by $I_{n}$. The $n$ dimensional column vector with all elements being 1 is denoted by $\mathcal{I}_{n}$.

\section{MODELING AND PRELIMINARIES}

\section{A. Rectifier Modeling}

The equivalent schematic diagram of the single-phase threelevel PWM rectifier device in CRH2 is shown in Fig. 1 where $u_{s}$ and $i_{s}$ are the grid side voltage and current respectively, $S_{i j}$, $i=1,2,3,4, j=a, b$ are the IGBT modules of bridges $a$ and $b$, respectively, $u_{1}$ and $u_{2}$ are the voltages of capacities $C_{1}$ and $C_{2}$ in dc-link side respectively, and $i_{l}$ is the load current. For simplifying analysis, the ideal switching functions $S_{j}, j=a, b$ are defined as in [21]. Suppose that there is no power loss and

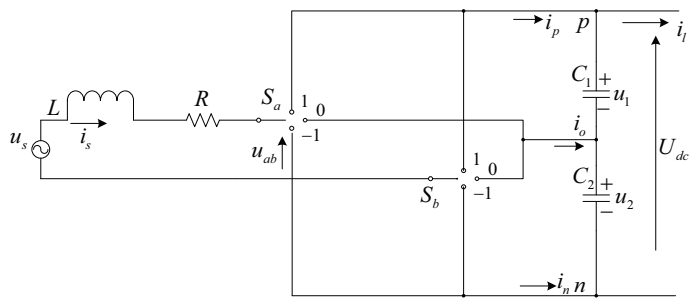

Fig. 1. Switch equivalent circuit

energy storage in IGBTs. Then it yields that

$$
u_{a b}=S_{1} u_{1}-S_{2} u_{2}, i_{p}=S_{1} i_{s} \text { and } i_{n}=S_{2} i_{s}
$$

where $S_{1}=\frac{S_{a}\left(S_{a}+1\right)-S_{b}\left(S_{b}+1\right)}{2}$ and $S_{2}=\frac{S_{a}\left(S_{a}-1\right)-S_{b}\left(S_{b}-1\right)}{2}$. Suppose that there is also no power loss and energy storage in the ac/dc/ac electrical traction system. Then the instantaneous powers are equal between the dc side and traction motors, that is

$$
\left(u_{1}+u_{2}\right) i_{l}=P_{m}
$$

where $P_{m}$ is the instantaneous power of traction motors.

It should be pointed out that the IGBT switching and the harmonics in grid side voltage $u_{s}$ result in harmonics in grid current $i_{s}$ and dc voltages $u_{1}$ and $u_{2}$. In this paper, these harmonics are considered as disturbances and uncertainties in the rectifier devices, and are represented by $\eta_{n}\left(u_{s}, i_{s}, u_{1}, u_{2}, \omega, t\right)=\operatorname{col}\left(\eta_{i_{s}}(\cdot), \eta_{u_{1}}(\cdot), \eta_{u_{2}}(\cdot)\right)$, which should be considered in fault diagnosis. From Fig. 1, on the basis of the Kirchhoff current and voltage principles, it has that

$$
\begin{aligned}
\frac{d i_{s}}{d t} & =\frac{1}{L}\left(u_{s}-R i_{s}-u_{a b}\right)+\eta_{i_{s}}\left(u_{s}, i_{s}, u_{1}, u_{2}, \omega\right), \\
\frac{d u_{1}}{d t} & =\frac{1}{C_{1}}\left(i_{p}-i_{l}\right)+\eta_{u_{1}}\left(u_{s}, i_{s}, u_{1}, u_{2}, \omega\right), \\
\frac{d u_{2}}{d t} & =\frac{1}{C_{2}}\left(-i_{n}-i_{l}\right)+\eta_{u_{2}}\left(u_{s}, i_{s}, u_{1}, u_{2}, \omega\right) .
\end{aligned}
$$

Thus, the state-space of the single-phase three-level rectifier device is obtained by

$$
\begin{aligned}
\dot{X} & =A_{n} X+g_{n}(X)+B_{n} U+\eta_{n}(X, U, \omega, t) \\
y & =X
\end{aligned}
$$

where $X=\operatorname{col}\left(i_{s}, u_{1}, u_{2}\right), U=u_{s}$ and $B_{n}=\operatorname{col}(1 / L, 0,0)$,

$$
A_{n}=\left[\begin{array}{ccc}
-\frac{R}{L} & \frac{-S_{1}}{L} & \frac{S_{2}}{L} \\
\frac{S_{1}}{C_{1}} & 0 & 0 \\
\frac{-S_{2}}{C_{2}} & 0 & 0
\end{array}\right] \text { and } g_{n}(X)=\left[\begin{array}{c}
0 \\
-\frac{P_{m}}{C_{1}\left(X_{2}+X_{3}\right)} \\
-\frac{P_{m}}{C_{2}\left(X_{2}+X_{3}\right)}
\end{array}\right] \text {. }
$$

\section{B. Incipient Sensor Fault Modeling}

An incipient sensor fault always develops in a continuous way that has been dicussed in [22], which can be modeled based on the following lemma.

Lemma 1. [23] For any piecewise continuous vector function $f: \mathcal{R}^{+} \rightarrow \mathcal{R}^{q}$, and a stable $q \times q$ matrix $A_{f}$, there always exists an input vector $\xi \in \mathcal{R}^{q}$ such that $\dot{f}=A_{f} f+\xi$.

For the dc voltage sensors, there are three fault modes: only sensor $u_{1}$ has incipient fault, only sensor $u_{2}$ has incipient fault and both sensors $u_{1}$ and $u_{2}$ have incipient faults simultaneously. From Lemma 1, these fault modes are modeled by

$$
\dot{f}^{i}=A_{f}^{i} f^{i}+D_{2}^{i} \Delta\left(u_{1}, u_{2}, U, \theta^{i}\right), f^{i}(0)=0, i=1,2,3
$$

where $A_{f}^{i}$ are Hurwitz matrices. The functions $\Delta\left(u_{1}, u_{2}, U, \theta^{i}\right)$ are continuous with small amplitude, which are used to describe the incipient faults. They are parameterized by $\theta^{i}$ nonlinearly. The faults considered are different from the existing linear parameterized faults as in [14]. It should be noted that $A_{f}^{i}$ are not design parameters. The unknown input distribution matrix $D_{2}^{i}, i=1,2,3$ are given by

$$
D_{2}^{1}=\left[\begin{array}{lll}
0 & 0 & 0 \\
0 & 1 & 0 \\
0 & 0 & 0
\end{array}\right], D_{2}^{2}=\left[\begin{array}{lll}
0 & 0 & 0 \\
0 & 0 & 0 \\
0 & 0 & 1
\end{array}\right], D_{2}^{3}=\left[\begin{array}{lll}
0 & 0 & 0 \\
0 & 1 & 0 \\
0 & 0 & 1
\end{array}\right] .
$$

Let $p$ and $N$ denote the number of sensors and number of possible fault modes in the rectifier device respectively. Then a set $\Sigma_{D}$ related to considered fault modes is defined by

$$
\Sigma_{D}=\left\{D_{2}^{i}, i=1, \cdots, N\right\} .
$$

The functions $\Delta\left(\cdot, \theta^{i}\right):=\operatorname{col}\left\{\delta\left(\cdot, \theta_{1}^{i}\right), \cdots, \delta\left(\cdot, \theta_{p}^{i}\right)\right\}, i=$ $1, \cdots, N$ where $\theta^{i}=\operatorname{col}\left(\theta_{1}^{i}, \cdots, \theta_{p}^{i}\right) \in \mathcal{R}^{p h}$ with $\theta_{j}^{i} \in R^{h}$, $j=1, \cdots, p$ represent the unknown fault parameter vectors, and are assumed to belong to known hypercubes $\Theta^{i}$, given by

$$
\theta^{i} \in \Theta^{i}=\left\{\theta^{i} \in \mathcal{R}^{p h} \mid \underline{\theta}_{j}^{i} \leq \theta_{j}^{i} \leq \bar{\theta}_{j}^{i}, j=1, \cdots, p h\right\} .
$$

where $\underline{\theta}_{j}^{i}$ and $\bar{\theta}_{j}^{i}$ are upper bound and lower bound of $\theta_{j}^{i}$ respectively. Therefore, the matrix $A_{f}^{i}$ and the unknown input 
$D_{2}^{i} \Delta\left(\cdot, \theta^{i}\right)$ characterize a class of incipient fault modes, in which the structure and time varying "magnitude" are parameterized by $A_{f}^{i}, D_{2}^{i}$ and $\theta^{i}$.

Remark 1. In [14], it is assumed that only one sensor fault occurs, which is can be applied to the case when multi faults occur simultaneously in practical systems. In this paper, multi incipient sensor faults isolation schemes are developed. The set $\Sigma_{D}$ in (10) contains multi incipient sensor faults mode in rectifier devices which will be isolated under certain conditions in this paper.

\section{Augmented System}

Let $x^{i}:=\operatorname{col}\left(X, f^{i}\right) \in \mathcal{R}^{n+p}$ where $n$ is the dimension of the rectifier. Inverter model (6)-(7) and fault models (8) can be represented in an augmented form as

$$
\begin{aligned}
\dot{x}^{i} & =A^{i} x^{i}+g\left(x^{i}\right)+B u+\eta\left(x^{i}, u, \omega, t\right)+D^{i} \Delta\left(y, u, \theta^{i}\right), \\
y & =\left[I_{p}, I_{p}\right] x^{i}, i=1, \cdots, N
\end{aligned}
$$

where $y$ is the actual measured signals, $u=U, A^{i}=$ $\operatorname{diag}\left(A_{n}, A_{f}^{i}\right), g\left(x^{i}\right)=\operatorname{col}\left(g_{n}\left(C_{x} x^{i}\right), 0\right)=\operatorname{col}\left(g_{n 0}\left(x^{i}\right), 0\right)$ with $C_{x}=\operatorname{diag}\left(I_{n}, 0\right), B=\operatorname{col}\left(B_{n}, 0\right), \eta(\cdot)=\operatorname{col}\left(\eta_{n}(\cdot), 0\right)$ and $D^{i}=\operatorname{col}\left(0, D_{2}^{i}\right)$. It should be pointed out that $D^{i}$ are not necessary full column rank, which are different from traditional papers such as in [7] and [8].

It is assumed throughout this paper that $\operatorname{rank}\left(D_{2}^{i}\right)=q_{i}, i=$ $1, \cdots, N$, which mean that there are $q_{i}$ elements 1 in diagonal of $D_{2}^{i}$. Since $D_{2}^{i}$ and $D_{2}^{j}(j \neq i)$ are diagonal matrices with elements 1 and 0 in diagonal, there exists one and only one orthogonal matrix $T_{0}^{i} \in \mathcal{R}^{p \times p}$ such that

$$
\left(T_{0}^{i}\right)^{T} D_{2}^{i} T_{0}^{i}=\left[\begin{array}{c|c}
0 & 0 \\
\hline 0 & D_{23}^{i i}
\end{array}\right]
$$

where $D_{23}^{i i}=I_{q_{i}}$. Then

$$
\left(T_{0}^{i}\right)^{T} D_{2}^{j} T_{0}^{i}=\left[\begin{array}{c|c}
D_{22}^{i j} & 0 \\
\hline 0 & D_{23}^{i j}
\end{array}\right]
$$

where $D_{22}^{i j} \in \mathcal{R}^{\left(p-q_{i}\right) \times\left(p-q_{i}\right)}$ and $D_{23}^{i j} \in \mathcal{R}^{q_{i} \times q_{i}}$ are diagonal matrices with only elements 1 and 0 in diagonals. Supposing that there are $d_{1}^{j}$ elements 1 in $D_{22}^{i j}$ and $d_{2}^{j}$ elements 1 in $D_{23}^{i j}$, respectively, $d_{1}^{j}+d_{2}^{j}=q_{j}$. Let

$$
T_{a}^{i}=\left[\begin{array}{c|c}
I_{n} & 0 \\
\hline I_{p} & \left(T_{0}^{i}\right)^{T}
\end{array}\right], T_{b}^{i}=T_{0}^{i} .
$$

Then

1) $z^{i}=T_{a}^{i} x^{i}=\operatorname{col}\left(z_{1}, z_{2}^{i}\right)=\operatorname{col}\left(X, X+\left(T_{0}^{i}\right)^{T} f^{i}\right)$ where $z_{1} \in \mathcal{R}^{n}$ and $z_{2}^{i} \in \mathcal{R}^{p}$,

2) $T_{a}^{i} A^{i}\left(T_{a}^{i}\right)^{-1}=\left[\begin{array}{c|c}A_{11} & 0 \\ \hline A_{21}^{i} & A_{22}^{i}\end{array}\right]=\left[\begin{array}{c|cc}A_{11} & \multicolumn{2}{|c}{0} \\ \hline A_{211}^{i} & \frac{A_{22}^{i 11}}{A_{212}^{i}} & A_{22}^{i 12} \\ \hline A_{22}^{i 21} & A_{22}^{i 22}\end{array}\right]=$ $\left[\begin{array}{cc}A_{n} & 0 \\ A_{n}-A_{f}^{i} & T_{0}^{i} A_{f}^{i}\left(T_{0}^{i}\right)^{T}\end{array}\right]$ where $A_{11}=A_{n}, A_{211}^{i} \in$ $\mathcal{R}^{\left(p-q_{i}\right) \times n}, A_{212}^{i} \in \mathcal{R}^{q_{i} \times n}, A_{22}^{i 11} \in \mathcal{R}^{\left(p-q_{i}\right) \times\left(p-q_{i}\right)}$ and $A_{22}^{i 22} \in \mathcal{R}^{q_{i} \times q_{i}}$,

3) $T_{a}^{i} D^{i} T_{b}^{i}=\left[\begin{array}{c|c}\multicolumn{2}{c}{0} \\ 0 & 0 \\ \hline 0 & D_{23}^{i i}\end{array}\right], T_{a}^{i} D^{j} T_{b}^{i}=\left[\begin{array}{c|c}2 & 0 \\ D_{22}^{i j} & 0 \\ \hline 0 & D_{23}^{i j}\end{array}\right]$,
4) $\left[I_{p}, F\right]\left(T_{a}^{i}\right)^{-1}=\left[\begin{array}{ll}0 & T_{0}^{i}\end{array}\right]$.

Accordingly, $T_{a}^{i} g(\cdot), T_{a}^{i} \eta(\cdot)$ and $T_{a}^{i} B$ can be obatined.

With matrix $\left(T_{b}^{i}\right)^{-1}$, the $j$ th $\Delta\left(\cdot, \theta^{j}\right), j=1, \cdots, N$ is assumed to be reordered as $\Delta^{i}\left(\cdot, \theta^{i j}\right)=\left(T_{b}^{i}\right)^{-1} \Delta\left(\cdot, \theta^{j}\right)$ where

$$
\begin{aligned}
\Delta^{i}\left(\cdot, \theta^{i j}\right) & =\operatorname{col}\left(\Delta_{1}^{i}\left(\cdot, \theta^{i j 1}\right), \Delta_{2}^{i}\left(\cdot, \theta^{i j 2}\right)\right), \\
\theta^{i j} & =\operatorname{col}\left(\theta^{i j 1}, \theta^{i j 2}\right)
\end{aligned}
$$

where

$$
\begin{aligned}
\Delta_{1}^{i}\left(\cdot, \theta^{i j 1}\right) & =\operatorname{col}\left(\delta\left(\cdot, \theta_{1}^{i j}\right), \cdots, \delta\left(\cdot, \theta_{p-q_{j}}^{i j}\right)\right) \\
\theta^{i j 1} & =\operatorname{col}\left(\theta_{1}^{i j}, \cdots, \theta_{p-q_{j}}^{i j}\right) \in \mathcal{R}^{\left(p-q_{j}\right) h}, \\
\Delta_{2}^{i}\left(\cdot, \theta^{i j 2}\right) & =\operatorname{col}\left(\delta\left(\cdot, \theta_{p-q_{j}+1}^{i j}\right), \cdots, \delta\left(\cdot, \theta_{p}^{i j}\right)\right), \\
\theta^{i j 2} & =\operatorname{col}\left(\theta_{p-q_{j}+1}^{i j}, \cdots, \theta_{p}^{i j}\right) \in \mathcal{R}^{q_{j} h} .
\end{aligned}
$$

Furthermore, for the $j$ th fault mode, the unknown fault parameter vector $\theta^{i j 1}$ in (20) and $\theta^{i j 2}$ in (22) are assumed to belong to known hypercubes $\Theta^{i j 1}$ and $\Theta^{i j 2}$ respectively, which can be obtained based on (11) and the matrix $T_{b}^{i}$.

Remark 2. It should be pointed out that different fault modes $j$ and transformations $T_{b}^{i}$ have different $\Theta^{i j 1}$ and $\Theta^{i j 2}$, which provide the capabilities to isolate different fault modes.

Assumption 1. The modeling uncertainties $\eta(\cdot)$ in (12) satisfies that $\forall t>0,\|\eta(\cdot)\| \leq \bar{\eta}$ where $\bar{\eta}$ is a known constant. Moreover, the nonlinear function $g_{n 0}\left(x^{i}\right)$ in (12) is uniformly Lipschitz, i.e. $\left\|g_{n 0}\left(x^{i}\right)-g_{n 0}\left(\hat{x}^{i}\right)\right\| \leq \mathscr{L}\left\|x^{i}-\hat{x}^{i}\right\|$ where $\mathscr{L}$ is the known Lipschitz constant.

Remark 3. In this paper, there is no constraint on the structure of uncertainties. The voltage and current harmonics are main sources of disturbances, and uncertain capacitances and inductances are the main sources of uncertainties in practical rectifier systems. In general, the injection expressions $\eta_{i_{s}}, \eta_{u_{1}}$ and $\eta_{u_{2}}$ in system (3)-(5) caused by harmonics and uncertain capacitances and inductances can be obtained based on rectifier working mechanism. Then the bounds $\bar{\eta}$ on uncertainties $\eta(\cdot)$ in (12) can be obtained based on specifications provided by manufacturers. Moreover, the historic statistical data can be used to help to estimate the bounds on external disturbances. $\nabla$

For the $i$ th fault mode, augmented system (12)-(13) is transformed to

$$
\begin{aligned}
\dot{z}_{1}= & A_{n} z_{1}+g_{n 0}\left(\left(T_{a}^{i}\right)^{-1} z^{i}\right)+B_{n} u+\eta_{n}(\cdot), \\
\dot{z}_{21}^{i}= & A_{211}^{i} z_{1}+A_{22}^{i 11} z_{21}^{i}+A_{22}^{i 12} z_{22}^{i}+g_{21}^{i}\left(\left(T_{a}^{i}\right)^{-1} z^{i}\right) \\
& +B_{21}^{i} u+\eta_{21}^{i}(\cdot), \\
\dot{z}_{22}^{i}= & A_{212}^{i} z_{1}+A_{22}^{i 21} z_{21}^{i}+A_{22}^{i 22} z_{22}^{i}+g_{22}^{i}\left(\left(T_{a}^{i}\right)^{-1} z^{i}\right) \\
& +B_{22}^{i} u+\eta_{22}^{i}(\cdot)+D_{23}^{i i} \Delta_{2}^{i}\left(y, u, \theta^{i i 2}\right), \\
y= & T_{0}^{i} z_{2}^{i}
\end{aligned}
$$

where $z_{2}^{i}=\operatorname{col}\left(z_{21}^{i}, z_{22}^{i}\right)$ with $z_{21}^{i}=\left[I_{p-q_{i}}, 0\right]\left(T_{0}^{i}\right)^{T} y \in$ $\mathcal{R}^{p-q_{i}}, z_{22}^{i}=\left[0, I_{q_{i}}\right]\left(T_{0}^{i}\right)^{T} y \in \mathcal{R}^{q_{i}}$. In (24) and (25), the functions $g_{21}^{i}$ and $g_{22}^{i}$, the matrices $B_{21}^{i}$ and $B_{22}^{i}$, and the uncertainties $\eta_{21}^{i}$ and $\eta_{22}^{i}$ are obtained from $T_{a}^{i}$. 
Fault detection is to detect the occurrence of faults, while fault isolation focuses on localization (classification) of different fault modes. In this paper, multi incipient sensor faults isolation schemes will be developed based on sliding mode technique and nonlinear parameterization adaptive estimation technique, which are completely different from [22].

\section{InCipient Sensor Fault Isolation Schemes}

\section{A. FIEs Design and Incipient Fault Isolation Decision Scheme}

Different from FIEs in [13], the following FIEs are constructed by combining sliding mode technique with nonlinear parameterization adaptive estimation technique. Suppose that the incipient sensor fault is detected at time $T_{d}$ when FIEs are activated. Each FIE corresponds to one potential fault mode. There are $N$ FIEs corresponding to $N$ potential fault modes. Denote $\hat{z}_{1}, \hat{z}_{21}^{s}$ and $\hat{z}_{22}^{s}$ as estimation of $z_{1}, z_{21}^{s}$ and $z_{22}^{s}$ respectively, and $e_{22}^{s}=z_{22}^{s}-\hat{z}_{22}^{s}$. The $s$ th FIE is designed as

$$
\begin{aligned}
\dot{\hat{z}}_{1}= & A_{n} \hat{z}_{1}+g_{n 0}\left(\left(T_{a}^{s}\right)^{-1} \hat{z}^{s}\right)+B_{n} u, \hat{z}_{1}\left(T_{d}\right)=0, \\
\dot{z}_{21}^{s}= & A_{211}^{s} \hat{z}_{1}+A_{22}^{s 11} \hat{z}_{21}^{s}+A_{22}^{s 12} z_{22}^{s}+g_{21}^{s}\left(\left(T_{a}^{s}\right)^{-1} \hat{z}^{s}\right) \\
& +K_{11}^{s}\left(z_{21}^{s}-\hat{z}_{21}^{s}\right)+B_{21}^{s} u+\bar{D}_{22}^{s s} \Delta_{1}^{s}\left(\cdot, \hat{\theta}^{s s 1}\right) \\
& +\nu_{21}^{s}+\nu_{22}^{s}, \hat{z}_{21}^{s}\left(T_{d}\right)=0, \\
\dot{\hat{z}}_{22}^{s}= & A_{212}^{s} \hat{z}_{1}+A_{22}^{s 21} z_{21}^{s}+A_{22}^{s 22} \hat{z}_{22}^{s}+K_{22}^{s}\left(z_{22}^{s}-\hat{z}_{22}^{s}\right) \\
& +\hat{A}_{22}^{s 22} \epsilon \operatorname{sat}\left(\frac{e_{22}^{s}}{\epsilon}\right)+g_{22}^{s}\left(\left(T_{a}^{s}\right)^{-1} \hat{z}^{s}\right)+B_{22}^{s} u \\
& +D_{23}^{s s} \Delta_{2}^{s}\left(\cdot, \hat{\theta}^{s s 2}\right)+D_{23}^{s s} \hat{\Lambda}_{2}^{s}\left(\cdot, \hat{\theta}^{s s 2}\right) \operatorname{sat}\left(\frac{e_{22}^{s}}{\epsilon}\right) \\
& -\Psi^{s}\left(z_{21}^{s}, z_{22}^{s}\right), \hat{z}_{22}^{s}\left(T_{d}\right)=0
\end{aligned}
$$

where $\hat{z}^{s} \triangleq \operatorname{col}\left(\hat{z}_{1},\left(T_{0}^{s}\right)^{T} y\right)$, the matrix $K_{11}^{s}=A_{22}^{s 11}-\hat{A}_{22}^{s 11}$ with $\hat{A}_{22}^{s 11}$ being symmetric negative definite, the matrix $\bar{D}_{22}^{s s}=$ $I_{p-q_{s}}$. The matrix $K_{22}^{s}=A_{22}^{s 22}-\hat{A}_{22}^{s 22}$ with $\hat{A}_{22}^{s 22}$ being symmetric negative definite and Metzler* where the positive system theory is used. The positive constant $\epsilon$ is chosen as a small scalar. The vector function $\hat{\Lambda}_{2}^{s}(\cdot)=\operatorname{diag}\left(\Lambda_{2}^{s}(\cdot)\right)$ where $\Lambda_{2}^{s}(\cdot)$ is given in (74) with $\alpha$ being chosen as $e_{22}^{s}$ (see Appendix). $\Psi^{s}(\cdot) \in \mathcal{R}^{q_{s}}$ will be determined later. The functions $\nu_{21}^{s}$ and $\nu_{22}^{s}$ are defined by

$$
\nu_{21}^{s}=m_{21}^{s}(\cdot) \operatorname{sgn}\left(z_{21}^{s}-\hat{z}_{21}^{s}\right), \nu_{22}^{s}=M_{22}^{s}(\cdot) \operatorname{sgn}\left(z_{21}^{s}-\hat{z}_{21}^{s}\right)
$$

where the scalar function $m_{21}^{s}(\cdot)$ and the diagonal matrix $M_{22}^{s}(\cdot)$ are determined later.

The vectors $\hat{\theta}^{s s 1}$ and $\hat{\theta}^{s s 2}$ are estimations of $\theta^{s s 1}$ and $\theta^{s s 2}$ respectively. The update laws, derived using Min-Max approach (see e.g., [19] and [24]), are proposed by

$$
\begin{aligned}
& \dot{\hat{\theta}}{ }^{s s 1}=\mathcal{P}_{\Theta^{s 1}}\left\{-\Gamma_{\theta^{s 1}} e_{21}^{s} W_{1}^{s}\left(\cdot, \hat{\theta}^{s s 1}\right)\right\}, \hat{\theta}^{s s 1}(0) \in \Theta^{s 1}, \\
& \dot{\hat{\theta}}^{s s 2}=\mathcal{P}_{\Theta^{s 2}}\left\{-\Gamma_{\theta^{s 2}} e_{22}^{s} W_{2}^{s}\left(\cdot, \hat{\theta}^{s s 2}\right)\right\}, \hat{\theta}^{s s 2}(0) \in \Theta^{s 2}
\end{aligned}
$$

where $\Gamma_{\theta^{s 1}}=\Gamma_{\theta^{s 1}}^{T}>0$ and $\Gamma_{\theta^{s 2}}=\Gamma_{\theta^{s 2}}^{T}>0$. The matrix function $W_{1}^{s}(\cdot)$ is given in (73) with $\alpha$ being chosen as $e_{21}^{s}$, and the matrix function $W_{2}^{s}(\cdot)$ is given in (74) with $\alpha$ being

*A real matrix is called Metzler matrix if all its off-diagonal entries are nonnegative. chosen as $e_{22}^{s}$ (See Appendix). In (31), the projection operator $\mathcal{P}_{\Theta^{s 1}}$ restricts the parameter estimation $\hat{\theta}^{s s 1}$ in $\Theta^{s 1}$. Also, $\mathcal{P}_{\Theta^{s 2}}$ in (32) restricts the parameter estimation $\hat{\theta}^{s s 2}$ in $\Theta^{s 2}$. In order to enter into sliding motion and guarantee the stability, the convex regions $\Theta^{s 1}$ and $\Theta^{s 2}$ are defined as

$$
\Theta^{s 1}=\bigcup_{i=1}^{N} \Theta^{s i 1}, \Theta^{s 2}=\Theta^{s s 2} .
$$

where $\Theta^{s i 1}$ and $\Theta^{s s 2}$ are obtained based on (11) and $T_{b}^{s}$.

This paper is different from the fault isolation schemes presented in [14] in that the adaptive threshold interval concept will be introduced later. Considering this, the incipient sensor fault mode isolation decision principle in this paper is presented as follows: if, for each $h \in\{1, \cdots, N\} \backslash\{s\}$, there exist some finite time $t^{h}>T_{d}$ and some $j \in\left\{1, \cdots, q_{h}\right\}$ such that $r_{j}^{h}(t) \notin\left[\underline{\varsigma}_{j}^{h}, \bar{\varsigma}_{j}^{h}\right]$, then the occurrence of the sth fault mode is concluded, where $r_{j}^{h}(t)$ represents the jth residual of the hth FIE, and $\left[\underline{\varsigma}_{j}^{h}, \bar{\varsigma}_{j}^{h}\right]$ is the corresponding adaptive threshold interval. The fault isolation time is defined as $T_{\text {isol }}^{s} \triangleq \max \left\{t^{h}, h \in\{1, \cdots, N\} \backslash\{s\}\right\}$.

\section{B. Stability Analysis}

Denote $e_{1}=z_{1}-\hat{z}_{1}$ and $e_{21}^{s}=z_{21}^{s}-\hat{z}_{21}^{s}$. Define the hyperplane sliding surfaces $\mathscr{S}^{s}$ for the $s$ th FIE as follows

$$
\mathscr{S}^{s}=\left\{\left(e_{1}, e_{21}^{s}\right) \mid e_{21}^{s}=0\right\}, s=1, \cdots, N .
$$

Note that

$$
\left(T_{a}^{s}\right)^{-1}\left(z^{s}-\hat{z}^{s}\right)=\left[\begin{array}{cc}
I_{n} & 0 \\
-T_{0}^{s} & T_{0}^{s}
\end{array}\right]\left[\begin{array}{c}
z_{1}-\hat{z}_{1} \\
0
\end{array}\right]=\left[\begin{array}{c}
I_{n} \\
-T_{0}^{s}
\end{array}\right]\left(z_{1}-\hat{z}_{1}\right) .
$$

From (23) and (27),

$$
\dot{e}_{1}=A_{n} e_{1}+g_{n 0}\left(\left(T_{a}^{s}\right)^{-1} z^{s}\right)-g_{n 0}\left(\left(T_{a}^{s}\right)^{-1} \hat{z}^{s}\right)+\eta_{n}(\cdot) .
$$

Thus, it can be obtained that $e_{1}=e^{A_{n} t} e_{1}\left(T_{d}\right)+$ $\int_{T_{d}}^{t} e^{A_{n}(t-\tau)}\left(g_{n 0}\left(\left(T_{a}^{s}\right)^{-1} z^{s}\right)-g_{n 0}\left(\left(T_{a}^{s}\right)^{-1} \hat{z}^{s}\right)+\eta_{n}(\cdot)\right) d \tau$.

In rectifier control systems, the objective is to ensure unity power factor operation and dc link voltage regulation. The inner loop of the rectifier device controls the input current $i_{s}$ using a classical PI controller, which is designed as a classical I-type system. When switching frequencies are high enough, the close-loop current in inner loop is first-order inertial element. The external loop of the rectifier device controls the dc link voltages $u_{1}$ and $u_{2}$ using a classical PI controller, which is designed as a classical II-type system. The closed-loop external loop closed-loop system is a minimum phase stable three-order damper system. Therefore, in rectifier control systems, the switching signals $S_{a}$ and $S_{b}$ are designed such that the switching system (6) is ISS (input-to-state stable) and $A_{n}$ is Hurwitz. Thus, from [25], there exist positive constants $k_{0}$ and $\lambda_{0}$ such that $\left\|e^{A_{n} t}\right\| \leq k_{0} e^{-\lambda_{0} t}, t \geq T_{d}$.

Suppose that there exists $\omega_{1}$ such that $\left\|e_{1}\left(T_{d}\right)\right\|=$ $\left\|z_{1}\left(T_{d}\right)\right\| \leq \omega_{1}$. Then by applying the Bellman-Gronwall lemma, it follows from Assumption 1 and (35) that $\left\|e_{1}(t)\right\|$ satisfies that

$$
\left\|e_{1}(t)\right\| \leq \chi(\cdot), t \geq T_{d}
$$

where $\chi(\cdot) \triangleq \chi\left(k_{0}, \bar{\eta}, \lambda_{0}, \mathscr{L}, T_{0}^{s}\right)$ which can be obtained from [25] and [13]. 
With the transformation matrices $T_{a}^{s}$ and $T_{b}^{s}$, in the presence of the $h$ th $(h \in\{1, \cdots, N\})$ fault mode, the unknown input of $z_{21}^{s}$ is described by $D_{22}^{s h} \Delta_{1}^{s}\left(y, u, \theta^{s h 1}\right)$. By comparing with (28), the error dynamic is obtained by

$$
\begin{aligned}
\dot{e}_{21}^{s}= & A_{211}^{s} e_{1}+\hat{A}_{22}^{s 11} e_{21}^{s}+g_{21}^{s}\left(\left(T_{a}^{s}\right)^{-1} z^{s}\right)-g_{21}^{s}\left(\left(T_{a}^{s}\right)^{-1} \hat{z}^{s}\right) \\
& -\nu_{21}^{s}+D_{22}^{s h} \Delta_{1}^{s}\left(\cdot, \theta^{s h 1}\right)-\bar{D}_{22}^{s s} \Delta_{1}^{s}\left(\cdot, \hat{\theta}^{s s 1}\right) \\
& +\eta_{21}(\cdot)-\nu_{22}^{s} .
\end{aligned}
$$

The following proposition is ready to be presented.

Proposition 1. Let $\kappa \geq \sup \left\|\theta^{s h 1}-\hat{\theta}^{s s 1}\right\|$ with $\theta^{s h 1} \in \Theta^{s h 1}$ and $\hat{\theta}^{s s 1}$ updated by (31). In the presence of the $h$ th fault mode, $h \in\{1, \cdots, N\}$, if the scalar function $m_{21}^{s}(y, u, t)$ and the diagonal matrix function $M_{22}^{s}\left(y, u, \hat{\theta}^{s s 1}\right)$ in (30) satisfy

$$
\begin{aligned}
m_{21}^{s}(\cdot) \geq & \left(\left\|A_{211}^{s}\right\|+\mathscr{L}\left(1+\left\|T_{0}^{s}\right\|\right)\right) \chi(\cdot) \\
& +\left\|W_{1}^{s}\left(\cdot, \hat{\theta}^{s s 1}\right)\right\| \kappa+\left\|\hat{\Lambda}_{1}^{s}\left(\cdot, \hat{\theta}^{s s 1}\right)\right\| \\
& +\mid \Delta_{1}^{s}\left(\cdot, \hat{\theta}^{s s 1}\right) \|+\bar{\eta}+\varpi, \\
M_{22}^{s}(\cdot)= & \hat{\Lambda}_{1}^{s}\left(\cdot, \hat{\theta}^{s s 1}\right), t \geq T_{d}
\end{aligned}
$$

where $\hat{\Lambda}_{1}^{s}(\cdot)=\operatorname{diag}\left(\Lambda_{1}^{s}(\cdot)\right), \Lambda_{1}^{s}(\cdot)$ and $W_{1}^{s}(\cdot)$ are given in (73) (see Appendix), then $e_{21}^{s}$ will enter into the hyperplane $\mathscr{S}^{s}$ given in (34) in finite time and will remain on it thereafter.

Proof: Let $V=\left(e_{21}^{s}\right)^{T} e_{21}^{s}$. It follows from (38) that $\dot{V}=$ $\dot{V}_{1}+\dot{V}_{2}+\dot{V}_{3}$ where $\dot{V}_{1}=\left(e_{21}^{s}\right)^{T}\left(\hat{A}_{22}^{s 11}+\left(\hat{A}_{22}^{s 11}\right)^{T}\right) e_{21}^{s}<0$ due to that $\hat{A}_{22}^{11 s}$ is symmetric negative definite,

$$
\begin{aligned}
\dot{V}_{2}= & \left(e_{21}^{s}\right)^{T}\left\{A_{211}^{s} e_{1}-D_{22}^{s h} W_{1}^{s}\left(\cdot, \hat{\theta}^{s s 1}\right)\left(\theta^{s h 1}-\hat{\theta}^{s s 1}\right)\right) \\
& \left.+g_{21}^{s}\left(\left(T_{a}^{s}\right)^{-1} z^{s}\right)-g_{21}^{s}\left(\left(T_{a}^{s}\right)^{-1} \hat{z}^{s}\right)-\eta_{21}^{s}(\cdot)-\nu_{21}^{s}\right\}
\end{aligned}
$$

and

$$
\begin{aligned}
\dot{V}_{3}= & \left(e_{21}^{s}\right)^{T}\left\{D_{22}^{s h} \Delta_{1}^{s}\left(\cdot, \theta^{s h 1}\right)-\bar{D}_{22}^{s s} \Delta_{1}^{s}\left(\cdot, \hat{\theta}^{s s 1}\right)\right. \\
& \left.+D_{22}^{s h} W_{1}^{s}\left(\cdot, \hat{\theta}^{s s 1}\right)\left(\theta^{s h 1}-\hat{\theta}^{s s 1}\right)-\nu_{22}^{s}\right\} .
\end{aligned}
$$

Note that $D_{22}^{s h}+\bar{D}_{22}^{s h}=I_{p-q_{s}}=\bar{D}_{22}^{s s}$. Then, $\dot{V}_{3}=\dot{V}_{31}+\dot{V}_{32}$ where

$$
\begin{gathered}
\dot{V}_{31}=\left(e_{21}^{s}\right)^{T}\left\{D_{22}^{s h} \Delta_{1}^{s}\left(\cdot, \theta^{s h 1}\right)-D_{22}^{s h} \Delta_{1}^{s}\left(\cdot, \hat{\theta}^{s s 1}\right)\right. \\
+D_{22}^{s h} W_{1}^{s}\left(\cdot, \hat{\theta}^{s s 1}\right)\left(\theta^{s h 1}-\hat{\theta}^{s s 1}\right) \\
\left.-D_{22}^{s h} \hat{\Lambda}_{1}^{s}\left(\cdot, \hat{\theta}^{s s 1}\right) \operatorname{sgn}\left(e_{21}^{s}\right)\right\}, \\
\dot{V}_{32}=-\left(e_{21}^{s}\right)^{T}\left\{\bar{D}_{22}^{s h} \Delta_{1}^{s}\left(\cdot, \hat{\theta}^{s s 1}\right)\right. \\
\left.+\bar{D}_{22}^{s h} \hat{\Lambda}_{1}^{s}\left(\cdot, \hat{\theta}^{s s 1}\right) \operatorname{sgn}\left(e_{21}^{s}\right)\right\} .
\end{gathered}
$$

From (69), (70) and (73), and choosing $\alpha=e_{21}^{s}$ in Appendix, it follows from the gain $M_{22}^{s}(\cdot)$ in (40) that $\Pi_{i}=$ $\left|e_{21 i}^{s}\right|\left(\operatorname{sgn}\left(e_{21 i}^{s}\right)\left(\delta\left(\cdot, \theta_{i}^{h}\right)-\delta\left(\cdot, \hat{\theta}_{i}^{s}\right)+w_{i}(\cdot)\left(\theta_{i}^{h}-\hat{\theta}_{i}^{s}\right)\right)-\lambda_{i}(\cdot)\right) \leq$ 0 . Then, $\dot{V}_{31}=\sum_{i=1}^{d_{1}^{h}} \Pi_{i}<0$. Due to the fact that $\kappa \geq \sup \left\|\theta^{s h 1}-\hat{\theta}^{s s 1}\right\|,\left\|D_{22}^{s h}\right\| \leq 1$ and $\left\|\bar{D}_{22}^{s h}\right\| \leq 1$, and also $\left\|\eta_{21}\right\|<\bar{\eta},\left\|g_{21}^{s}\left(\left(T_{a}^{s}\right)^{-1} z^{s}\right)-g_{21}^{s}\left(\left(T_{a}^{s}\right)^{-1} \hat{z}^{s}\right)\right\| \leq$ $\mathscr{L}\left(1+\left\|T_{0}^{s}\right\|\right)\left\|e_{1}\right\|$, the gain $m_{21}^{s}(\cdot)$ in (39) ensures that $\dot{V}_{2}+\dot{V}_{32} \leq-\varpi\left\|e_{21}\right\|$. Therefore, $\dot{V} \leq-\varpi\left\|e_{21}\right\| \leq-\varpi V^{1 / 2}$, which implies that the reachability condition is satisfied.

Hence, the conclusion follows.

Remark 4. It should be pointed out that these isolation schemes require that all sliding motions take place earlier than faults occur. Compared with abrupt faults, incipient sensor faults in rectifiers usually take long time to cause system failures. In addition, the time taken to reach sliding surfaces (34) can be reduced by adjusting $\varpi$ and $\hat{z}_{21}^{s}(0)$ to ensure that the sliding motions occur at the very initial stage. Therefore, the developed results can be applied to a majority of cases in reality.

\section{Adaptive Threshold Interval}

Based on the fault mode isolation principle presented in Section III-A, the $s$ th fault isolation residual $r^{s}$ is required to be sensitive not only to the sth fault mode, but also to the $h$ th fault mode with $h \in\{1, \cdots, N\} \backslash\{s\}$, which is different from [14] since sliding mode is introduced in FIEs (27)-(29). Therefore, a tuning error $e_{22 \epsilon}^{s}=e_{22}^{s}-\epsilon \operatorname{sat}\left(\frac{e_{22}^{s}}{\epsilon}\right)+T^{s} z_{21}^{s}$ is defined as the isolation residual, where the constant matrix $T^{s} \in \mathcal{R}^{q_{s} \times\left(p-q_{s}\right)}$ is chosen such that $T^{s} D_{22}^{s h} \neq 0$ when $D_{22}^{s h} \neq$ 0 for the $h$ th fault mode to guarantee that $e_{22 \epsilon}^{s}$ is sensitive to $D_{22}^{s h} \Delta_{1}^{s}(\cdot)$.

Accordingly, the $\Psi^{s}(\cdot)$ in (29) is given by

$$
\begin{aligned}
\Psi^{s}(\cdot)= & T^{s} A_{211}^{s} \hat{z}_{1}+T^{s} A_{22}^{s 11} z_{21}^{s}+T^{s} A_{22}^{s 12} z_{22}^{s} \\
& +T^{s} g_{21}^{s}\left(\left(T_{a}^{s}\right)^{-1} \hat{z}^{s}\right)-\hat{A}_{22}^{s 22} T^{s} z_{21}^{s} .
\end{aligned}
$$

For the $s$ th fault mode, when $e_{22}^{s} \geq \epsilon, \dot{e}_{22 \epsilon}^{s}=\dot{e}_{22}^{s}+T^{s} \dot{z}_{21}^{s}$. By substituting (45) to (29) and comparing with (25), the error dynamic is obtained by

$$
\begin{aligned}
\dot{e}_{22 \epsilon}^{s}= & \left(A_{212}^{s}+T^{s} A_{211}^{s}\right) e_{1}+\hat{A}_{22}^{s 22} e_{22 \epsilon}^{s} \\
& +\left(g_{22}^{s}+T^{s} g_{21}^{s}\right)\left(\left(T_{a}^{s}\right)^{-1}\left(z^{s}-\hat{z}^{s}\right)\right) \\
& +\left(\eta_{22}^{s}+T^{s} \eta_{21}^{s}\right)(\cdot)+D_{23}^{s s} \Delta_{2}^{s}\left(\cdot, \theta^{s s 2}\right) \\
& -D_{23}^{s s} \Delta_{2}^{s}\left(\cdot, \hat{\theta}^{s s 2}\right)-D_{23}^{s s} \bar{\Lambda}_{2}^{s}\left(\cdot, \hat{\theta}^{s s 2}\right) .
\end{aligned}
$$

Also when $e_{22}^{s} \leq-\epsilon, \dot{e}_{22 \epsilon}^{s}=\dot{e}_{22}^{s}+T^{s} \dot{z}_{21}^{s}$,

$$
\begin{aligned}
\dot{e}_{22 \epsilon}^{s}= & \left(A_{212}^{s}+T^{s} A_{211}^{s}\right) e_{1}+\hat{A}_{22}^{s 22} e_{22 \epsilon}^{s} \\
& +\left(g_{22}^{s}+T^{s} g_{21}^{s}\right)\left(\left(T_{a}^{s}\right)^{-1}\left(z^{s}-\hat{z}^{s}\right)\right) \\
& +\left(\eta_{22}^{s}+T^{s} \eta_{21}^{s}\right)(\cdot)+D_{23}^{s s} \Delta_{2}^{s}\left(\cdot, \theta^{s s 2}\right) \\
& -D_{23}^{s s} \Delta_{2}^{s}\left(\cdot, \hat{\theta}^{s s 2}\right)+D_{23}^{s s} \underline{\Lambda}_{2}^{s}\left(\cdot, \hat{\theta}^{s s 2}\right) .
\end{aligned}
$$

Remark 5. The estimation error $\operatorname{col}\left(e_{21}^{s}, e_{22}^{s}\right)$ is chosen as isolation residual in [13] and [14] while only $e_{22 \epsilon}^{s}$ is selected as residual in this paper, which and the characteristic that $e_{21}^{s}=$ 0 when sliding motion occurs facilitate the design of more proper adaptive thresholds to improve isolability.

According to (46) and (47), the adaptive threshold interval is defined as $\left[\varsigma^{s}, \bar{\varsigma}^{s}\right]$ where

$\dot{\bar{\zeta}}=\left(\Upsilon_{1}+\bar{\Upsilon}_{2}\right) \mathcal{I}_{q_{s}}+\hat{A}_{22}^{s 22} \bar{\varsigma}^{s}, \quad \dot{\varsigma}^{s}=-\left(\Upsilon_{1}+\underline{\Upsilon}_{2}\right) \mathcal{I}_{q_{s}}+\hat{A}_{22}^{s 22} \underline{\varsigma}^{s}$

where $\Upsilon_{1}=\left(\left\|A_{212}^{s}+T^{s} A_{211}^{s}\right\|+\mathscr{L}\left(1+\left\|T_{0}^{s}\right\|\right)\right) \chi(t)+(1+$ $\left.\left\|T^{s}\right\|\right) \bar{\eta}>0, \bar{\Upsilon}_{2}=\left\|\bar{W}_{2}^{s}\left(\cdot, \hat{\theta}^{s s 2}\right)\right\|\left\|\tilde{\theta}^{s s 2}\right\|>0$ and $\underline{\Upsilon}_{2}=$ $\left\|\underline{W}_{2}^{s}\left(\cdot, \hat{\theta}^{s s 2}\right)\right\|\left\|\tilde{\theta}^{s s 2}\right\|>0$.

Under the initial condition that $\bar{\varsigma}^{s}\left(T_{d}\right) \geq 0$ and $\varsigma^{s}\left(T_{d}\right) \leq 0$, it has that $\bar{\varsigma}^{s}(t) \geq 0$ and $\underline{\varsigma}^{s}(t) \leq 0, \forall t>T_{d}$ because $\hat{A}_{22}^{s 22}$ is Metzler.

Define two errors $\bar{e}_{22 \epsilon}^{s}=\bar{\varsigma}^{s}-e_{22 \epsilon}^{s}$ when $e_{22}^{s} \geq \epsilon$, and $\underline{e}_{22 \epsilon}^{s}=e_{22 \epsilon}^{s}-\underline{\varsigma}^{s}$ when $e_{22}^{s} \leq-\epsilon$, respectively. Assume that 
$\underline{\varsigma}^{s}\left(T_{d}\right) \leq e_{22 \epsilon}^{s}\left(T_{d}\right) \leq \bar{\varsigma}^{s}\left(T_{d}\right), \bar{e}_{22 \epsilon}^{s}\left(T_{d}\right) \geq 0$ and $\underline{e}_{22 \epsilon}^{s}\left(T_{d}\right) \geq 0$. Then

$$
\begin{aligned}
& \dot{\bar{e}}_{22 \epsilon}^{s}=\hat{A}_{22}^{s 22} \bar{e}_{22 \epsilon}^{s}+\bar{\phi}, \bar{e}_{22 \epsilon}^{s}\left(T_{d}\right) \geq 0, \\
& \underline{\dot{e}}_{22 \epsilon}^{s}=\hat{A}_{22}^{s 22} \underline{e}_{22 \epsilon}^{s}+\underline{\phi}, \underline{e}_{22 \epsilon}^{s}\left(T_{d}\right) \geq 0
\end{aligned}
$$

where $\bar{\phi}$ and $\phi$ can be obtained based on (46), (47) and (48).

From (71) and (72) in Appendix with $\alpha=e_{22}^{s}$, it can be obtained that when $e_{22}^{s} \geq \epsilon, \bar{\phi} \geq 0$, and when $e_{22}^{s} \leq-\epsilon$, $\phi \geq 0$.

Consider when one of the component $\bar{e}_{22 \epsilon i}^{s}$ of $\bar{e}_{22 \epsilon}^{s}$ in (49) is equal to zero for the first time at $t=t_{1}>T_{d}$. Then,

$$
\dot{\bar{e}}_{22 \epsilon i}^{s}\left(t_{1}\right)=\sum_{j=1}^{q_{s}} \hat{A}_{22 i j}^{s 2} \bar{e}_{22 \epsilon j}^{s}\left(t_{1}\right)+\bar{\phi}_{j}
$$

where $\hat{A}_{22 i j}^{s 22}$ represent the $i$ th row and $j$ th column of $\hat{A}_{22}^{s 22}$. At $t=t_{1}, \bar{e}_{22 \epsilon i}^{s}\left(t_{1}\right)=0, \bar{e}_{22 \epsilon j}^{s}\left(t_{1}\right) \geq 0, j \neq i$ and $\bar{\phi}_{j} \geq 0$. From the fact that $\hat{A}_{22}^{s 22}$ is Metzler, $\hat{A}_{22 i j}^{s 22}>0, i \neq j$, then $\dot{\bar{e}}_{22 \epsilon i}^{s}\left(t_{1}\right) \geq 0$, which implies that $\bar{e}_{22 \epsilon i}^{s}$ will stay nonnegative. Finally $\bar{e}_{22 \epsilon i}^{s}$ remains nonnegative for any time $t \geq T_{d}$. Therefore, $\bar{e}_{22 \epsilon}^{s} \geq 0, \forall t>T_{d}$. Using the same analysis, the result that $\underline{e}_{22 \epsilon}^{s} \geq 0, \forall t>T_{d}$ can be obtained.

Then, the following proposition is ready to be presented.

Proposition 2. If $0 \leq e_{22 \epsilon}^{s}\left(T_{d}\right) \leq \bar{\varsigma}^{s}\left(T_{d}\right)$, then $\bar{\varsigma}^{s}(t) \geq 0$, and $e_{22 \epsilon}^{s}(t) \leq \bar{\varsigma}^{s}(t), \forall t>T_{d}$, else if $\underline{\varsigma}^{s}\left(T_{d}\right) \leq e_{22 \epsilon}^{s}\left(T_{d}\right) \leq 0$, $\varsigma^{s}(t) \leq 0, \varsigma^{s}(t) \leq e_{22 \epsilon}^{s}(t), \forall t>T_{d}$. Furthermore, if $\underline{\varsigma}^{s}\left(T_{d}\right) \leq$ $e_{22 \epsilon}^{s}\left(T_{d}\right) \leq \bar{\varsigma}^{s}\left(T_{d}\right)$, then $\underline{s}^{s}(t) \leq e_{22 \epsilon}^{s}(t) \leq \bar{\varsigma}^{s}(t), \forall t>T_{d}$.

Proof: The result is obtained directly from the analysis above and the proof is omitted here.

It should be noted that the estimation error $\tilde{\theta}^{s s 2}$ is used in (48). Since $\theta^{s s 2}$ is unknown, $\bar{\varsigma}^{s}$ and $\varsigma^{s}$ cannot be used in the design directly. The projection adaptive law proposed in (32) ensures that $\left\|\tilde{\theta}^{s s 2}\right\|$ is bounded by a known constant $\kappa_{1}$. Therefore, in the fault isolation scheme, $\left\|\tilde{\theta}^{s s 2}\right\|$ in (48) can be replaced by $\kappa_{1}$ in practical design.

\section{Incipient Sensor Fault Isolability Analysis}

For the $r$ th FIE $(r=1, \cdots, N)$, there exist two variables $\bar{\vartheta}^{r r}$ and $\underline{\vartheta}^{r r}$ satisfying that

$$
\begin{aligned}
& \dot{\bar{\vartheta}}^{r r}=\hat{A}_{22}^{r 22} \bar{\vartheta}^{r r}+D_{23}^{r r}\left(\Delta_{2}^{r}\left(\cdot, \hat{\theta}^{r r 2}\right)+\bar{\Lambda}_{2}^{r}\left(\cdot, \hat{\theta}^{r r 2}\right)\right), \\
& \dot{\underline{\vartheta}}^{r r}=\hat{A}_{22}^{r 22} \underline{\vartheta}^{r r}+D_{23}^{r r}\left(\Delta_{2}^{r}\left(\cdot, \hat{\theta}^{r r 2}\right)+\underline{\Lambda}_{2}^{r}\left(\cdot, \hat{\theta}^{r r 2}\right)\right)
\end{aligned}
$$

where $\bar{\vartheta}^{r r}\left(T_{d}\right)=0$ and $\underline{\vartheta}^{r r}\left(T_{d}\right)=0$. Also for the $s$ th fault mode and the $r$ th FIE, there exists a variable $\zeta^{r s}$ satisfying that $\zeta^{r s}\left(T_{d}\right)=0$,

$$
\dot{\zeta}^{r s}=\hat{A}_{22}^{r 22} \zeta^{r s}+T^{r} D_{22}^{r s} \Delta_{1}^{r}\left(\cdot, \theta^{r s 1}\right)+D_{23}^{r s} \Delta_{2}^{r}\left(\cdot, \theta^{r s 2}\right) .
$$

To measure the different effects between the two fault modes, the two functions between the $s$ th fault mode and the $h$ th fault mode are defined by

$$
\bar{J}^{r s}=\zeta^{r s}-\bar{\vartheta}^{r r}, \underline{J}^{r s}=\zeta^{r s}-\underline{\vartheta}^{r r} .
$$

Remark 6. From a qualitative point of view, $\bar{J}^{r s}$ and $\underline{J}^{r s}$ can be interpreted as a filtered version of the difference between the actual fault functions $T^{r} D_{22}^{r s} \Delta_{1}^{r}\left(y, u, \theta^{r s 1}\right)+$
$D_{23}^{r s} \Delta_{2}^{r}\left(y, u, \theta^{r s 2}\right)$ and its estimation $D_{23}^{r r} \Delta_{2}^{s}\left(y, u, \hat{\theta}^{r r 2}\right)$ associated with the $r$ th FIE whose structure does not match the actual $s$ th fault mode. The functions given in (55), defined as the ability of the $r$ th FIE to match the $s$ th fault mode, provide a quantitive measure of the difference between the $s$ th fault mode and the $r$ th fault mode.

Then the following theorem is ready to be presented.

Theorem 1. Consider the FIEs described by (27)-(29). Suppose that the $s$ th fault mode occurs at time $t=T_{0}$ which is detected at time $t=T_{d}$. The $s$ th fault mode is isolable if for each $r \in\{1, \cdots, N\} \backslash\{s\}$, there exist certain time $t^{r}>T_{d}$ and some $j \in\left\{1, \cdots, q_{r}\right\}$ such that the functions $\bar{J}_{j}^{r s}$ or $\underline{J}_{j}^{r s}$ satisfy that

$$
\bar{J}_{j}^{r s}\left(t^{r}\right) \leq-\overline{\mathcal{F}}_{j}^{r s}-\bar{\delta}_{j}^{r}, \underline{J}_{j}^{r s}\left(t^{r}\right) \geq \underline{\mathcal{F}}_{j}^{r s}-\underline{\varsigma}_{j}^{r}
$$

where $\bar{J}_{j}^{r s}$ and $\underline{J}_{j}^{r s}$ are the $j$ th component of $\bar{J}^{r s}$ and $\underline{J}^{r s}$ respectively, $\bar{\delta}_{j}^{r}$ and $\underline{\varsigma}_{j}^{r}$ are the $j$ th component of $\bar{\varsigma}^{r}$ and $\underline{\varsigma}^{r}$ respectively, $\underline{\mathcal{F}}_{j}^{r s}$ and $\underline{\mathcal{F}}_{j}^{r s}$ will be given later.

Proof: When the sth fault mode occurs, the $r$ th FIE dynamic is described by

$$
\begin{aligned}
\dot{e}_{22}^{r}= & A_{212}^{r} e_{1}+\hat{A}_{22}^{r 22}\left(e_{22}^{r}-\epsilon \operatorname{sat}\left(\frac{e_{22}^{r}}{\epsilon}\right)\right)+g_{22}^{r}\left(\left(T_{a}^{r}\right)^{-1} z^{r}\right) \\
& -g_{22}^{r}\left(\left(T_{a}^{r}\right)^{-1} \hat{z}^{r}\right)+\eta_{22}^{r}(\cdot)+D_{23}^{r s} \Delta_{2}^{r}\left(\cdot, \theta^{r s 2}\right) \\
& -D_{23}^{r r} \Delta_{2}^{r}\left(\cdot, \hat{\theta}^{r r 2}\right)-D_{23}^{r r} \Lambda_{2}^{r}\left(\cdot, \hat{\theta}^{r r 2}\right) \operatorname{sat}\left(\frac{e_{22}^{r}}{\epsilon}\right)-\Psi(\cdot) . \quad(57)
\end{aligned}
$$

From the definition of the residual $e_{22 \epsilon}^{r}=e_{22}^{r}-\epsilon \operatorname{sat}\left(\frac{e_{22}^{r}}{\epsilon}\right)+$ $T^{r} z_{21}^{r}$, the dynamics of $e_{22 \epsilon}^{r}$ can be obtained based on (57).

Let $\bar{e}_{22 \epsilon}^{r s}=e_{22 \epsilon}^{r}+\bar{\vartheta}^{r r}-\zeta^{r s}$. It follows from the dynamics of $e_{22 \epsilon}^{r}$ that

$$
\begin{aligned}
\dot{\bar{e}}_{22 \epsilon}^{r s}= & \left(A_{212}^{r}+T^{r} A_{211}\right) e_{1}+\hat{A}_{22}^{r 22} \bar{e}_{22 \epsilon}^{r s}+\left(T^{r} g_{21}^{r}+g_{22}^{r}\right) \\
& \left(\left(T_{a}^{r}\right)^{-1} z^{r}-\left(T_{a}^{r}\right)^{-1} \hat{z}^{r}\right)+\left(T^{r} \eta_{21}+\eta_{22}^{r}\right)(\cdot) .
\end{aligned}
$$

Since $\hat{A}_{22}^{r 22}$ is Hurwitz, using the Bellman-Gronwall lemma and the similar reasoning as that used in (37), there exists a bank of positive time functions $\overline{\mathcal{F}}_{j}^{r s}(t), j=1, \cdots, q_{s}$ such that

$$
-\overline{\mathcal{F}}_{j}^{r s}(t) \leq \bar{e}_{22 \epsilon j}^{r s} \leq \overline{\mathcal{F}}_{j}^{r s}(t)
$$

where $\bar{e}_{22 \epsilon j}^{r s}$ represents the $j$ th component of $\bar{e}_{22 \epsilon}^{r s}$ in (58).

Let $\underline{e}_{22 \epsilon}^{r s}=e_{22 \epsilon}^{r}+\underline{\vartheta}^{r r}-\zeta^{r s}$ when $e_{22}^{r} \leq-\epsilon$. Similar with (59), there exists a bank of positive time functions $\underline{\mathcal{F}}_{j}^{r s}(t)$ such that

$$
-\underline{\mathcal{F}}_{j}^{r s}(t) \leq \underline{e}_{22 \epsilon j}^{r s} \leq \underline{\mathcal{F}}_{j}^{r s}(t)
$$

where $\underline{e}_{22 \epsilon j}^{r s}$ represents the $j$ th component of $\underline{e}_{22 \epsilon}^{r s}$.

To isolate the $s$ th fault mode, it requires that at least one component $e_{22 \epsilon j}^{r}, j=1, \cdots, q_{r}$ of $e_{22 \epsilon}^{r}, r \in\{1, \cdots, N\} \backslash\{s\}$ runs out of the adaptive threshold interval $\left[\underline{\varsigma}_{j}^{r}, \bar{\varsigma}_{j}^{r}\right]$. Since, when $e_{22 \epsilon}^{r}>0, e_{22 \epsilon}^{r}=\bar{e}_{22 \epsilon}^{r s}-\bar{J}^{r s}$, and when $e_{22 \epsilon}^{r}<0$, $e_{22 \epsilon}^{r}=\underline{e}_{22 \epsilon}^{r s}-\underline{J}^{r s}$, then

$$
e_{22 \epsilon j}^{r}=\bar{e}_{22 \epsilon j}^{r s}-\bar{J}_{j}^{r s} \geq \bar{\varsigma}_{j}^{r} \text { or } e_{22 \epsilon j}^{r}=\underline{e}_{22 \epsilon j}^{r s}-\underline{J}_{j}^{r s} \leq \underline{\varsigma}_{j}^{r} .
$$

Hence, eqs. (56) are obtained, and the result follows. 
Remark 7. It can be seen from (56) in Theorem 1 that the matrices $A_{f}^{s}$ and $A_{f}^{r}$ do not affect isolation ability directly because (56) does not contain $A_{f}^{s}$ and $A_{f}^{r}$. From the analysis in Section III-B, it obtains that $A_{f}^{i}$ does not affect the stability of sliding mode and adaptive FIEs. Therefore, matrices $A_{f}^{i}$ in (4) are chosen by considering not only the practical characteristics of incipient rectifier voltage sensor faults, but also the enlargement of the differences between $\theta^{i}$.

Remark 8. It is worthy to point out that it is challenging to develop multi sensor faults isolation schemes only using sliding mode technique (see e.g., [9]). In this paper, the nonlinear parameterization adaptive estimation technique is introduced to propose novel multi incipient sensor faults isolation schemes which are quite different from single sensor fault isolation schemes in [14].

\section{VERIFICATION}

In this section, simulation based on the model (6)-(7) and experiment based on TDCS-FIB (traction and driving control system-fault injection benchmark) will be presented. The TDCS-FIB is programmed based on SimPower System toolbox to simulate common faults that may occur on $\mathrm{CRH} 2$ by Central South University Fault Injection Team, which is outside the red rectangle frame in Fig. 2. The designed incipient voltage sensor fault isolation schemes are included in the red rectangle frame in Fig. 2. In both simulation and exper-

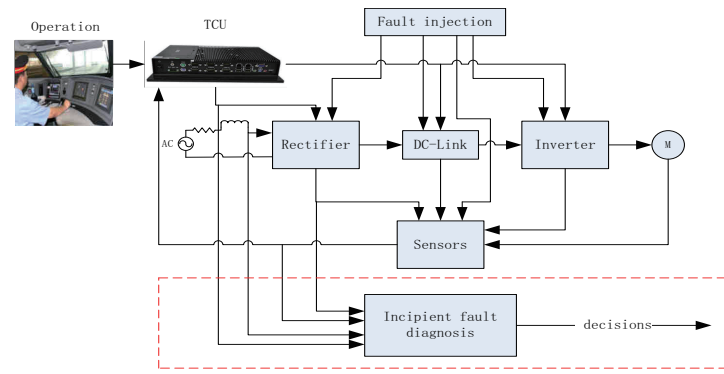

Fig. 2. Schematic diagrams of TDCS-FIB and fault isolation schemes.

iment, the railway is supposed to work at a fixed velocity. Then the instantaneous power $P_{m}$ is fixed. The parameters in model (6)-(7) and values of the electrical components in the rectifier device in Fig. 2 are given by Table I. Thus, the matrices

TABLE I

PARAMETERS.

\begin{tabular}{lcc}
\hline \hline Parameter & Value & Unit \\
\hline$P_{m}$ & 800 & $k W$ \\
$R$ & 0.34 & $\Omega$ \\
$L$ & $2.2 \times 10^{-3}$ & $H$ \\
$C_{1}$ & $1.6 \times 10^{-3}$ & $F$ \\
$C_{2}$ & $1.6 \times 10^{-3}$ & $F$ \\
$u_{s}$ & $1500 \sqrt{2} \sin (314 t)$ & $V$ \\
\hline \hline
\end{tabular}

in (6)-(7) are given by $A_{n}=\left[\begin{array}{ccc}-154.55 & -454.55 S_{1} & 454.55 S_{2} \\ 62.50 S_{1} & 0 & 0 \\ -62.50 S_{2} & 0 & 0\end{array}\right]$, $B_{n}=\left[\begin{array}{c}454.55 \\ 0 \\ 0\end{array}\right]$ and $g_{n}(X)=\left[\begin{array}{c}0 \\ -\frac{5 \times 10^{6}}{X_{2}+X_{3}} \\ -\frac{5 \times 10^{6}}{X_{2}+X_{3}}\end{array}\right]$ where $S_{i}, i=1,2$ are generated by TCU (traction control unit) module.
Consider the 3 -order harmonic of grid side current $i_{s}$ and parameter uncertainties $\Delta_{R}=0.02 \Omega, \Delta_{L}=0.2 \times$ $10^{-4} \mathrm{H}$ and $\Delta_{C}=2 \times 10^{-4} \mathrm{~F}$ in this paper. Then $\eta_{i s}=$ $\frac{H_{i_{s}} i_{s}}{\sqrt{2} \cos (\omega t)} \sin (3 \omega t-\varphi)+\Delta_{1 i_{s}} i_{s}+\Delta_{2 i_{s}} u_{1}+\Delta_{3 i_{s}} u_{2}, \eta_{u_{1}}=$ $\Delta_{1 u_{1}} i_{s}$ and $\eta_{u_{2}}=\Delta_{1 u_{2}} i_{s}$ with $H_{i_{s}}=100, \omega=314, \Delta_{1 i_{s}}=$ $7.6145, \Delta_{2 i_{s}}=4.0950, \Delta_{3 i_{s}}=-4.0950, \Delta_{1 u_{1}}=-0.7716$ and $\Delta_{1 u_{2}}=0.7716$. Thus, $\bar{\eta}$ in Assumption 1 is obtained by

$$
\bar{\eta} \geq\left\|\left[\begin{array}{ccc}
\frac{H_{i_{s}} \sin (3 \omega t-\varphi)}{\sqrt{2} \cos (\omega t)}+\Delta_{1 i_{s}} & \Delta_{2 i_{s}} & \Delta_{3 i_{s}} \\
\Delta_{1 u_{1}} & 0 & 0 \\
\Delta_{1 u_{2}} & 0 & 0
\end{array}\right]\right\|\|X\| .
$$

Note that $i_{s} \in[0,500]$ and $u_{1}, u_{2} \in[1000,1500]$ in CRH2. Then the constant bound $\bar{\eta}$ can be obtained. Using differential mean value theorem presented in [26], the Lipschitz constant of $g_{n}(X)$ in Assumption 1 is $\mathscr{L}=1.58$.

The base function $\delta(\cdot)$ used to construct $\Delta(\cdot)$ in (8) is given by

$$
\delta(\cdot)=\zeta\left(u_{1}, u_{2}, t\right) \theta^{2}
$$

where $\zeta\left(u_{1}, u_{2}, t\right)=0.01\left(u_{1} \sin (314 t)+u_{2} \sin (314 t+\pi / 3)+\right.$ $\left.\left(u_{1}+u_{2}\right) \sin (314 t+2 \pi / 3)\right)$. The distribution matrices in (9) and the basis function $\delta(\cdot)$ are all used in the sequel simulation and experiment.

\section{A. Simulation}

In the 1st fault mode, the incipient fault is modeled by

$$
\dot{f}^{1}=A_{f}^{1} f^{1}+D_{2}^{1} \Delta\left(u_{1}, u_{2}, \theta^{1}\right), f^{1}(0)=0
$$

where $A_{f}^{1}=-100 I_{3}$. It is assumed that fault parameters $\theta_{1}^{1}$, $\theta_{2}^{1}$ and $\theta_{3}^{1}$ in $\Delta\left(\cdot, \theta^{1}\right)=\operatorname{col}\left(\delta\left(\cdot, \theta_{1}^{1}\right), \delta\left(\cdot, \theta_{2}^{1}\right), \delta\left(\cdot, \theta_{3}^{1}\right)\right)$ belong to the intervals that $\theta_{1}^{1}=0, \theta_{2}^{1} \in[0,100], \theta_{3}^{1}=0$. In the 2 nd fault mode, the incipient faults is modeled by

$$
\dot{f}^{2}=A_{f}^{2} f^{2}+D_{2}^{2} \Delta\left(u_{1}, u_{2}, \theta^{2}\right), f^{2}(0)=0
$$

where $A_{f}^{2}=-20 I_{3}$ and the intervals of fault parameters $\theta_{1}^{2}$, $\theta_{2}^{2}$ and $\theta_{3}^{2}$ in $\Delta\left(\cdot, \theta^{2}\right)$ are given by $\theta_{1}^{2}=0, \theta_{2}^{2}=0$ and $\theta_{3}^{2} \in$ $[0,50]$. In the $3 \mathrm{rd}$ fault mode, the incipient faults is modeled by

$$
\dot{f}^{3}=A_{f}^{3} f^{3}+D_{2}^{3} \Delta\left(u_{1}, u_{2}, \theta^{3}\right), f^{3}(0)=0
$$

where $A_{f}^{3}=-50 I_{3}$ and the intervals of fault parameters $\theta_{1}^{3}$, $\theta_{2}^{3}$ and $\theta_{3}^{3}$ in $\Delta\left(\cdot, \theta^{3}\right)$ are given by $\theta_{1}^{3}=0, \theta_{2}^{3} \in[0,60]$ and $\theta_{3}^{3} \in[0,80]$.

Suppose that the fault parameters $\theta_{1}^{3}=0, \theta_{2}^{3}=0, \theta_{3}^{3}=0$ before $0.15 \mathrm{~s}$, and $\theta_{1}^{3}=0, \theta_{2}^{3}=40, \theta_{3}^{3}=60$ after $0.15 \mathrm{~s}$. As comparison, the fault isolation method in [13] and [14] is used to isolate this incipient sensor fault mode firstly. The simulation results of the 1st FIE constructed based on [13] and [14] are shown in Figs. 3 and 4. Since that It can be seen from Fig. 3 that the incipient fault parameter estimation lines are not convergent, which means that the adaptive laws in [13] and [14] can not be used to estimate incipient faults parameterized nonlinearly efficiently. Furthermore, it is clear from Figs. 4 that none of the isolation residuals exceeds their adaptive thresholds, which is conflicting with the isolation requirement in [13] and [14]. Thus, there is no need to show the simulation results of the 2ed FIE and no isolation decision can be made based on the principles provided in [13] and [14]. 


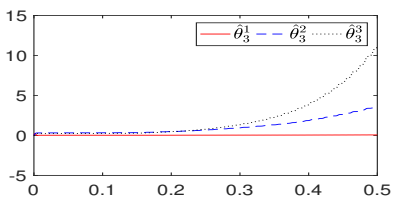

Fig. 3. Parameter estimation lines in the 1st and 2nd FIEs.

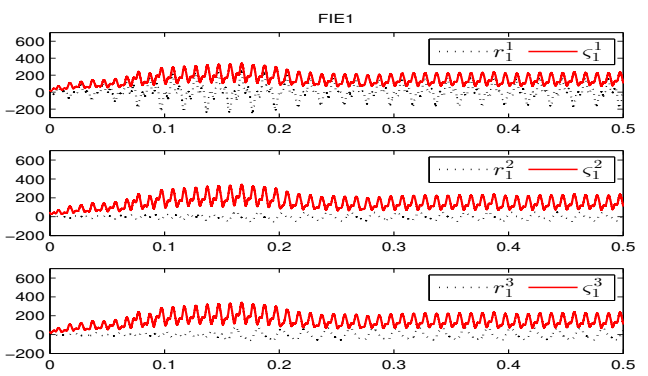

Fig. 4. Residuals of the 1rd FIE (dashed and black lines) and corresponding adaptive thresholds (solid and red lines).

The reason is that multi sensor faults cases are not considered in these two paper.

Then, the multi incipient faults isolation schemes developed in this paper are constructed. Corresponding to the three fault modes, there are three groups coordinate transformation matrices for three FIEs. The 1st group is described by

$$
T_{a}^{1}=\left[\begin{array}{llll}
I_{3} & 0 & 0 & 0 \\
0 & 1 & 0 & 0 \\
0 & 0 & 0 & 1 \\
0 & 0 & 1 & 0
\end{array}\right], T_{b}^{1}=\left[\begin{array}{lll}
1 & 0 & 0 \\
0 & 0 & 1 \\
0 & 1 & 0
\end{array}\right],
$$

For the 2nd and 3rd groups, $T_{a}^{2}=T_{a}^{3}=I_{6}$ and $T_{b}^{2}=T_{b}^{3}=I_{3}$. Two important schemes in FIEs are constructing adaptive laws $\dot{\hat{\theta}}^{s s 1}$ and $\dot{\hat{\theta}}^{s s 2}$ for nonlinear incipient fault parameters in (31) and (32), and selecting gains $m_{21}^{s}(\cdot)$ and $M_{22}^{s}(\cdot)$ to ensure sliding motion in Proposition 1. In two schemes, $\bar{\lambda}_{i}(\cdot), \underline{\lambda}_{i}(\cdot)$, $\bar{w}_{i}(\cdot)$ and $\underline{w}_{i}(\cdot), i=1,2,3$ are used to construct $W_{1}^{i}(\cdot), \Lambda_{1}^{i}(\cdot)$, $W_{2}^{i}(\cdot)$ and $\Lambda_{2}^{i}(\cdot)$. Based on Lemma 2, these terms for the 3rd incipient fault mode (65) with nonlinear parameterization (62) in the 1st FIE are expressed by $\bar{\lambda}_{1}(\cdot)=0, \bar{\lambda}_{2}(\cdot)=0, \bar{\lambda}_{3}(\cdot)=$ $0, \bar{w}_{1}(\cdot)=0, \bar{w}_{2}(\cdot)=-100 \delta(\cdot), \bar{w}_{3}(\cdot)=-100 \delta(\cdot)$, $\underline{\lambda}_{1}(\cdot)=0, \underline{\lambda}_{2}(\cdot)=-\delta(\cdot)\left(\hat{\theta}_{3}^{3}\right)^{2}+100 \delta(\cdot) \hat{\theta}_{3}^{3}, \underline{\lambda}_{3}(\cdot)=$ $-\delta(\cdot)\left(\hat{\theta}_{2}^{3}\right)^{2}+100 \delta(\cdot) \hat{\theta}_{2}^{3}, \underline{w}_{1}(\cdot)=0, \underline{w}_{2}(\cdot)=-2 \delta(\cdot) \hat{\theta}_{3}^{3}$ and $\underline{w}_{3}(\cdot)=-2 \delta(\cdot) \hat{\theta}_{2}^{3}$. The expressions of those terms in the 2 nd and the 3rd FIEs are similar to these in the 1st FIE, which are omitted here due to space limitation. Then the adaptive laws in (31) and (32), and gains in (39) and (40) can be constructed. Thus, based on (27)-(29), these three FIEs corresponding to the three incipient sensor fault modes can be easily constructed.

The estimation lines of incipient fault parameters in three developed FIEs are shown in Fig. 5. It can be seen that all the estimations lines are bounded. The incipient sensor fault isolation results are shown in Figs. 6 and 7. It can be seen from Fig. 6 that $r^{1}$ in the 1st FIE exceeds the lower bound $\varsigma^{1}$ at $t_{1}$, and $r^{2}$ in the 2st FIE exceeds $\varsigma^{2}$ at $t_{2}$ as well, while $r_{1}^{3}$ and $r_{2}^{3}$ are both stay in their threshold intervals $\left[\varsigma_{1}^{3}, \bar{\varsigma}_{1}^{3}\right]$ and $\left[\underline{\varsigma}_{2}^{3}, \bar{\varsigma}_{2}^{3}\right]$ all the time respectively in Fig. 7. As a result, based
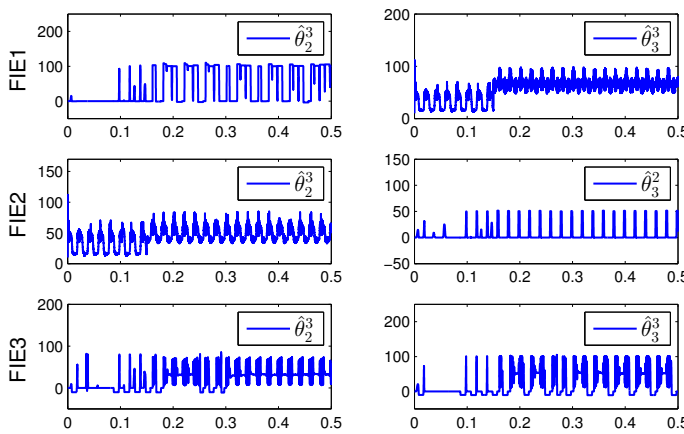

Fig. 5. Parameter estimation lines in the 1st, 2nd and 3rd FIEs.

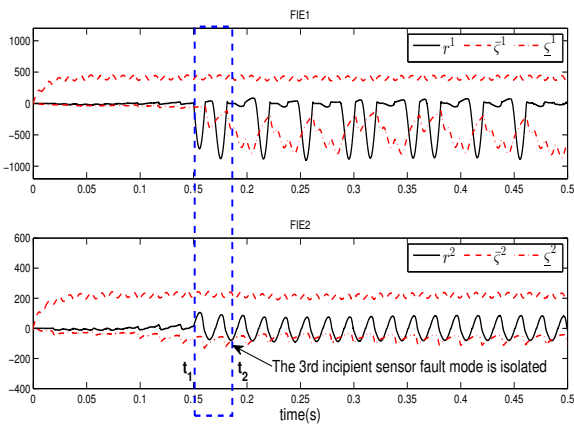

Fig. 6. Residuals of the 1st and 2nd FIE (solid and black lines) and corresponding adaptive threshold intervals (dashed and red lines).
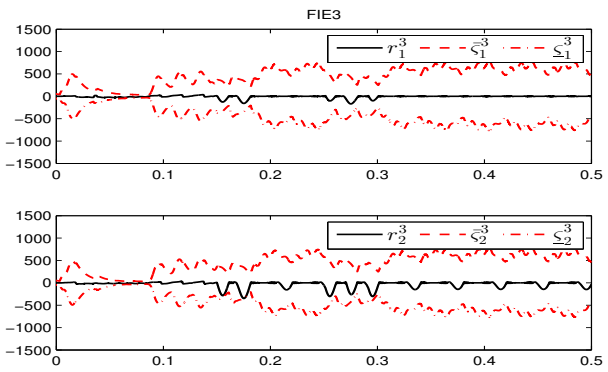

Fig. 7. Residuals of the 3rd FIE (solid and black lines) and corresponding adaptive threshold intervals (dashed and red lines).

on the isolation principle developed in this paper, the decision that the 3rd incipient sensor fault mode occurs at time instant $t_{2}$ is made.

\section{B. Experiment}

The TDCS-FIB in Fig. 2 can effectively simulate some common faults occurring in the electric traction and driving system of high-speed railway, which provides a good platform to study the fault diagnosis issue for electrical traction systems. The two voltage sensors in DC-Link module in Fig. 2 are both injected incipient faults provided by fault expression (65) with incipient sensor fault parameters $\theta_{1}^{3}, \theta_{2}^{3}$ and $\theta_{3}^{3}$ given as in the simulation part. The developed incipient sensor fault isolation schemes are established and added to the traction system in the way presented in the red rectangle frame in Fig. 2. 


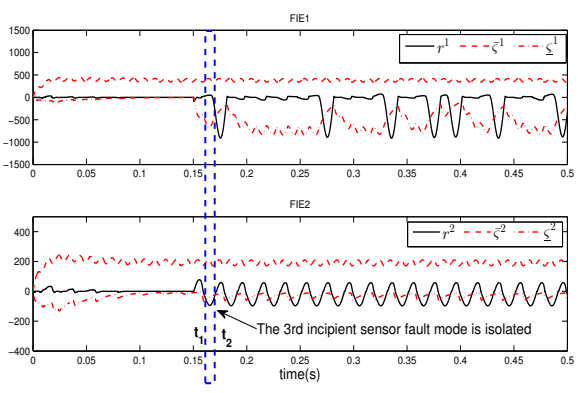

Fig. 8. Residuals of the 1st and 2nd FIE (solid and black lines) and corresponding adaptive threshold intervals (dashed and red lines).
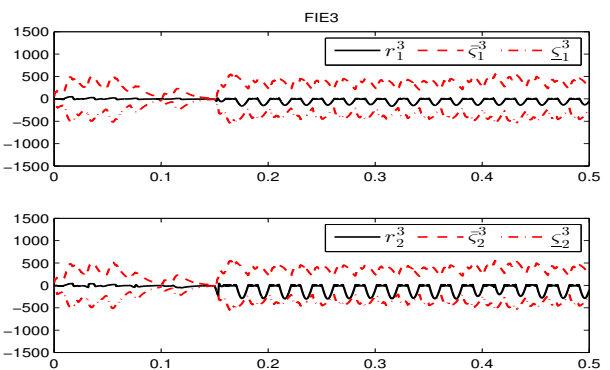

Fig. 9. Residuals of the 3rd FIE (solid and black lines) and corresponding adaptive threshold intervals (dashed and red lines).

The response curves in experiment in Fig. 8 and Fig. 9 are similar with those in simulation in Figs. 6 and 7. It can be seen from Fig. 8 and Fig. 9 that $r^{2}$ in the 2nd FIE exceeds $\left[\varsigma^{2}, \bar{\varsigma}^{2}\right]$ at $t_{1}$, and $r^{1}$ in the 1 st FIE exceeds $\left[\varsigma^{1}, \bar{\varsigma}^{1}\right]$ at $t_{2}$. However, residuals $r_{1}^{3} \in\left[\underline{s}_{1}^{3}, \bar{\varsigma}_{1}^{3}\right]$ and $r_{2}^{3} \in\left[\underline{\varsigma}_{2}^{3}, \bar{\varsigma}_{2}^{3}\right]$ all the time. Therefore, based on the isolation principle developed in this paper, it can make the decision that both voltage sensors have incipient faults after time instant $t_{2}$.

\section{CONCLUSION}

This paper has proposed a dc voltage incipient sensor fault isolation scheme for single-phase three-level rectifier devices in high-speed railway electrical traction systems. A novel incipient fault isolation method has been developed by combining sliding mode technique with nonlinear parametrization adaptive estimation technique. In the proposed method, novel residuals and adaptive threshold intervals have been presented to isolate different fault modes. The isolability has been studied and the sufficient isolable conditions have been derived by the defined functions. Based on TDCS-FIB, simulation and experiment results have demonstrated that the proposed method is effective and practicable.

\section{APPENDIX}

Lemma 2. For any bounded continuous known scalar function $f(\phi, \theta)$ with $\phi$ being known and $\theta \in \Theta \subset \mathcal{R}^{h}$, and any given $\hat{\theta} \in \Theta$, there exist vector function $w(\phi, \hat{\theta}) \in \mathcal{R}^{h}$ and scalar function $\lambda(\phi, \hat{\theta})$ such that

$$
J(w(\cdot), \theta)-\lambda(\cdot) \leq 0
$$

where $J(w(\cdot), \theta)=\operatorname{sign}(\alpha)(f(\phi, \theta)-f(\phi, \hat{\theta})+w(\cdot)(\theta-\hat{\theta}))$ with $\alpha$ being known.

Proof: Refer [19] and [24].

According to Lemma 2, for any $\delta\left(\cdot, \theta_{r}^{j}\right), \theta_{r}^{j} \in \mathcal{R}^{h}, r=$ $1, \cdots, p, j=1, \cdots, N$, there exist row function vector $\underline{w}_{r}\left(\cdot, \hat{\theta}_{r}^{j}\right) \in \mathcal{R}^{h}$ and scalar function $\underline{\lambda}_{r}\left(\cdot, \hat{\theta}_{r}^{j}\right)$ such that

$$
\delta\left(\cdot, \theta_{r}^{j}\right)-\delta\left(\cdot, \hat{\theta}_{r}^{j}\right)+\underline{w}_{r}\left(\cdot, \hat{\theta}_{r}^{j}\right)\left(\theta_{r}^{j}-\hat{\theta}_{r}^{j}\right)+\underline{\lambda}_{r}\left(\cdot, \hat{\theta}_{r}^{j}\right)>0,
$$

and row function vector $\bar{w}_{r}\left(\cdot, \hat{\theta}_{r}^{j}\right) \in \mathcal{R}^{h}$ and scalar function $\bar{\lambda}_{r}\left(\cdot, \hat{\theta}_{r}^{j}\right)$ such that

$$
\delta\left(\cdot, \theta_{r}^{j}\right)-\delta\left(\cdot, \hat{\theta}_{r}^{j}\right)+\bar{w}_{r}\left(\cdot, \hat{\theta}_{r}^{j}\right)\left(\theta_{r}^{j}-\hat{\theta}_{r}^{j}\right)-\bar{\lambda}_{r}\left(\cdot, \hat{\theta}_{r}^{j}\right)<0 .
$$

Given $\hat{\theta}^{i j 1} \in \Theta^{i j 1}$, for $\Delta_{1}^{i}\left(\cdot, \theta^{i j 1}\right)$ in (19), there exist diagonal matrix functions $\bar{W}_{1}^{i}\left(\cdot, \hat{\theta}^{i j 1}\right)$ and $\underline{W}_{1}^{i}\left(\cdot, \hat{\theta}^{i j 1}\right)$ and vector functions $\bar{\Lambda}_{1}^{i}\left(\cdot, \hat{\theta}^{i j 1}\right)$ and $\underline{\Lambda}_{1}^{i}\left(\cdot, \hat{\theta}^{i j 1}\right)$ such that

$\Delta_{1}^{s}\left(\cdot, \theta^{i j 1}\right)-\Delta_{1}^{s}\left(\cdot, \hat{\theta}^{i j 1}\right)+\underline{W}_{1}^{s}(\cdot)\left(\theta^{i j 1}-\hat{\theta}^{i j 1}\right)+\underline{\Lambda}_{1}^{s}(\cdot)>0$,

$\Delta_{1}^{s}\left(\cdot, \theta^{i j 1}\right)-\Delta_{1}^{s}\left(\cdot, \hat{\theta}^{i j 1}\right)+\bar{W}_{1}^{s}(\cdot)\left(\theta^{i j 1}-\hat{\theta}^{i j 1}\right)-\bar{\Lambda}_{1}^{s}(\cdot)<0$

where $\bar{W}_{1}^{i}\left(\cdot, \hat{\theta}^{i j 1}\right)=\operatorname{diag}\left(\bar{w}_{r}\left(\cdot, \hat{\theta}^{i j 1}\right)\right), \underline{W}_{1}^{i}\left(\cdot, \hat{\theta}^{i j 1}\right)=$ $\operatorname{diag}\left(\underline{w}_{r}\left(\cdot, \hat{\theta}^{i j 1}\right)\right), \quad \bar{\Lambda}_{1}^{i}\left(\cdot, \hat{\theta}^{i j 1}\right)=\operatorname{col}\left(\bar{\lambda}_{r}\left(\cdot, \hat{\theta}^{i j 1}\right)\right)$ and $\underline{\Lambda}_{1}^{i}\left(\cdot, \hat{\theta}^{i j 1}\right)=\operatorname{col}\left(\underline{\lambda}_{r}\left(\cdot, \hat{\theta}^{i j 1}\right)\right), r=1, \cdots, p-q_{i}$.

Also, given $\hat{\theta}^{i j 2} \in \Theta^{i j 2}$ for $\Delta_{2}^{i}\left(\cdot, \theta^{i j 2}\right)$ in (21), there exist diagonal matrix functions $\bar{W}_{2}^{i}\left(\cdot, \hat{\theta}^{i j 2}\right)$ and $\underline{W}_{2}^{i}\left(\cdot, \hat{\theta}^{i j 2}\right)$ and vector functions $\bar{\Lambda}_{2}^{i}\left(\cdot, \hat{\theta}^{i j 2}\right)$ and $\underline{\Lambda}_{2}^{i}\left(\cdot, \hat{\theta}^{i j 2}\right)$ such that

$\Delta_{2}^{i}\left(\cdot, \theta^{i j 2}\right)-\Delta_{2}^{i}\left(\cdot, \hat{\theta}^{i j 2}\right)+\underline{W}_{2}^{i}(\cdot)\left(\theta^{i j 2}-\hat{\theta}^{i j 2}\right)+\underline{\Lambda}_{2}^{i}(\cdot)>0$

$\Delta_{2}^{i}\left(\cdot, \theta^{i j 2}\right)-\Delta_{2}^{i}\left(\cdot, \hat{\theta}^{i j 2}\right)+\bar{W}_{2}^{i}(\cdot)\left(\theta^{i j 2}-\hat{\theta}^{i j 2}\right)-\bar{\Lambda}_{2}^{i}(\cdot)<0$

where $\bar{W}_{2}^{i}=\operatorname{diag}\left(\bar{w}_{r}\left(\cdot, \hat{\theta}^{i j 2}\right)\right), \underline{W}_{2}^{i}=\operatorname{diag}\left(\underline{w}_{r}\left(\cdot, \hat{\theta}^{i j 2}\right)\right)$, $\bar{\Lambda}_{2}^{i}=\operatorname{col}\left(\bar{\lambda}_{r}\left(\cdot, \hat{\theta}^{i j 2}\right)\right)$ and $\Lambda_{2}^{i}=\operatorname{col}\left(\underline{\lambda}_{r}\left(\cdot, \hat{\theta}^{i j 2}\right)\right), r=p-$ $q_{i}+1, \cdots, p$. The $W_{1}^{i}\left(\cdot, \hat{\theta}^{i j 1}\right)$ and $\Lambda_{1}^{i}\left(\cdot, \hat{\theta}^{i j 1}\right)$ are defined as

$$
\left\{\begin{array}{l}
W_{1}^{i}(\cdot, \cdot)=\bar{W}_{1}^{i}(\cdot, \cdot), \Lambda_{1}^{i}(\cdot, \cdot)=\bar{\Lambda}_{1}^{i}(\cdot, \cdot), \alpha>0, \\
W_{1}^{i}(\cdot, \cdot)=\underline{W}_{1}^{i}(\cdot, \cdot), \Lambda_{1}^{i}(\cdot, \cdot)=\underline{\Lambda}_{1}^{i}(\cdot, \cdot), \alpha<0,
\end{array}\right.
$$

and $W_{2}^{i}\left(\cdot, \hat{\theta}^{i j 2}\right)$ and $\Lambda_{2}^{i}\left(\cdot, \hat{\theta}^{i j 2}\right)$ are defined as

$$
\left\{\begin{array}{l}
W_{2}^{i}(\cdot, \cdot)=\bar{W}_{2}^{i}(\cdot, \cdot), \Lambda_{2}^{i}(\cdot, \cdot)=\bar{\Lambda}_{2}^{i}(\cdot, \cdot), \alpha>0, \\
W_{2}^{i}(\cdot, \cdot)=\underline{W}_{2}^{i}(\cdot, \cdot), \Lambda_{2}^{i}(\cdot, \cdot)=\underline{\Lambda}_{2}^{i}(\cdot, \cdot), \alpha<0 .
\end{array}\right.
$$

\section{REFERENCES}

[1] Z. He, H. Hu, Y. Zhang, and S. Gao, "Harmonic resonance assessment to traction power-supply system considering train model in china highspeed railway," IEEE Transactions on Power Delivery, vol. 29, DOI 10.1109/TIE.2016.2610400, no. 4, pp. 1735-1743, Oct. 2014.

[2] A. Youssef, E. Khil, and S. Khojet, "State observer-based sensor fault detection and isolation, and fault-tolerant control of a single-phase pwm rectifier for electric railway traction," IEEE Transactions on Power Electronics, vol. 28, DOI 10.1109/TPEL.2013.2257862, no. 12, pp. 5842-5853, Dec. 2013.

[3] S. Yang, D. Xiang, A. Bryant, P. Mawby, L. Ran, and P. Tavner "Condition monitoring for device reliability in power electronic converters: A review," IEEE Transactions on Power Electronics, vol. 25, DOI 10.1109/TPEL.2010.2049377, no. 11, pp. 2734-2752, May. 2010.

[4] A. M. Imam, "Condition monitoring of electrolytic capacitors for power electronics applications," Ph.D. dissertation, Georgia Institute of Technology, 2007.

[5] P. M. Frank, "Fault diagnosis in dynamic systems using analytical and knowledge-based redundancy: A survey and some new results," Automatica, vol. 26, DOI 10.1016/0005-1098(90)90018-D, no. 3, pp. 459-474, Dec. 1990. 
[6] J. Liu, W. Luo, X. Yang, and L. Wu, "Robust model-based fault diagnosis for pem fuel cell air-feed system," IEEE Transactions on Industrial Electronics, vol. 63, DOI 10.1109/TIE.2016.2535118, no. 5, pp. 32613270, Feb. 2016.

[7] C. Edwards, S. K. Spurgeon, and R. J. Patton, "Sliding mode observers for fault detection and isolation," Automatica, vol. 36, DOI 10.1016/S0005-1098(99)00177-6, no. 4, pp. 541-553, Apr. 2000.

[8] X. G. Yan and C. Edwards, "Nonlinear robust fault reconstruction and estimation using a sliding mode observer," Automatica, vol. 43, DOI 10.1016/j.automatica.2007.02.008, no. 9, pp. 1605-1614, Sep. 2007.

[9] W. Chen and W. Saif, "Observer-based strategies for actuator fault detection, isolation and estimation for certain class of uncertain nonlinear systems," IET Control Theory \& Applications, vol. 1, DOI 10.1049/ietcta:20060408, no. 6, pp. 1672-1680, Nov. 2007.

[10] Z. Y. Sun, L. R. Xue, and K. Zhang, "A new approach to finitetime adaptive stabilization of high-order uncertain nonlinear system," Automatica, vol. 58, DOI 10.1016/j.automatica.2015.05.005, pp. 60-66, Aug. 2015.

[11] Y. Zhang, G. Tao, and M. Chen, "Adaptive neural network based control of noncanonical nonlinear systems," IEEE Transactions on Neural Networks and Learning Systems, vol. 27, DOI 10.1109/TNNLS.2015.2461001, no. 9, pp. 1864-1877, Aug. 2016.

[12] B. Jiang, M. Staroswiecki, and V. Cocquempot, "Fault accommodation for nonlinear dynamic systems," IEEE Transactions on Automatic Control, vol. 51, DOI 10.1109/TAC.2006.878732, no. 9, pp. 1578 - 1583, Sep. 2006

[13] X. Zhang, M. M. Polycarpou, and T. Parisini, "Fault diagnosis of a class of nonlinear uncertain systems with lipschitz nonlinearities using adaptive estimation," Automatica, vol. 46, DOI 10.1016/j.automatica.2009.11.014, no. 2, pp. 290-299, Feb. 2010.

[14] X. Zhang, "Sensor bias fault detection and isolation in a class of nonlinear uncertain systems using adaptive estimation," IEEE Transactions on Automatic Control, vol. 56, DOI 10.1109/TAC.2011.2112471, no. 5, pp. 1220-1226, May. 2011.

[15] X. G. Yan and C. Edwards, "Adaptive sliding-mode-observer-based fault reconstruction for nonlinear systems with parametric uncertainties," IEEE Transactions on Industrial Electronics, vol. 55, DOI 10.1109/TIE.2008.2003367, no. 11, pp. 4029-4036, Nov. 2008.

[16] H. Alwi and C. Edwards, "An adaptive sliding mode differentiator for actuator oscillatory failure case reconstruction," Automatica, vol. 49, DOI 10.1016/j.automatica.2012.11.042, no. 2, pp. 642-651, Feb. 2013.

[17] X. G. Yan, C. Edwards, and S. K. Spurgeon, "Decentralised robust sliding mode control for a class of nonlinear interconnected systems by static output feedback," Automatica, vol. 40, DOI 10.1016/j.automatica.2003.10.025, pp. 613-620, Apr. 2004.

[18] J. Liu, S. Vazquez, L. Wu, A. Marquez, H. Gao, and L. G. Franquelo, "Extended state observer-based sliding-mode control for three-phase power converters," IEEE Transactions on Industrial Electronics, vol. 64, DOI 10.1109/TIE.2016.2610400, no. 1, pp. 22-31, Feb. 2017.

[19] A. Annaswamy, F. Skantze, and A. Loh, "Adaptive control of continuous time systems with convex/concave parametrization," Automatica, vol. 34, DOI 10.1016/S0005-1098(97)00159-3, no. 1, pp. 33-49, Jan. 1998.

[20] J. L. Gouzé, A. Rapaport, and M. Z. Hadj-Sadok, "Interval observers for uncertain biological systems," Ecological modelling, vol. 133, DOI 10.1016/S0304-3800(00)00279-9, no. 1, pp. 45-56, Aug. 2000.

[21] W. S. Song, X. Y. Feng, and C. Xiong, "A neutral point voltage regulation method with svpwm control for single-phase three-level npc converters," in 2008 IEEE Vehicle Power and Propulsion Conference, DOI 10.1109/VPPC.2008.4677472, pp. 1 - 4, Sept. 2008.

[22] K. Zhang, B. Jiang, X. G. Yan, and Z. Mao, "Sliding mode observer based incipient sensor fault detection with application to high-speed railway traction device," ISA transactions, vol. 63, DOI 10.1016/j.isatra.2016.04.004, pp. 49-59, Jul. 2016.

[23] M. Saif and Y. Guan, "A new approach to robust fault detection and identification," IEEE Transactions on Aerospace and Electronic Systems, vol. 29, DOI 10.1109/7.220921, no. 3, pp. 685-695, Jul. 1993.

[24] A. P. Loh, A. M. Annaswamy, and F. P. Skantze, "Adaptation in the presence of a general nonlinear parameterization: an error model approach," IEEE Transation on Automatic Control, vol. 44, DOI 10.1109/9.788531, no. 9, pp. 1634-1652, Septemper 1999.

[25] X. G. Yan and C. Edwards, "Robust sliding mode observer-based actuator fault detection and isolation for a class of nonlinear sys- tems," International Journal of Systems Science, vol. 39, DOI 10.1080/00207720701778395, no. 4, pp. 349-359, Feb. 2008.

[26] A. Zemouche, M. Boutayeb, and G. I. Bara, "Observer design for nonlinear systems: an approach based on the differential mean value theorem," Proceedings of the 44th IEEE Conference on Decision and Control, DOI 10.1109/CDC.2005.1583180, pp. 6353-6358, Jan. 2005.

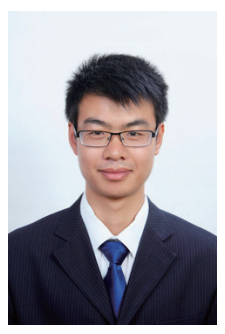

Kangkang Zhang received the B.Sc. degree in automatic control from Henan University of Technology Henan, China in 2008, M.Sc. degree in control theory and control engineering from Northeasten University, Shenyang, China in 2014. He is now a Ph.D. student at the College of Automation Engineering, Nanjing University of Aeronautics and Astronautics. His research interests cover incipient fault diagnosis under disturbances, fault-tolerant control, sliding mode control and adaptive control.

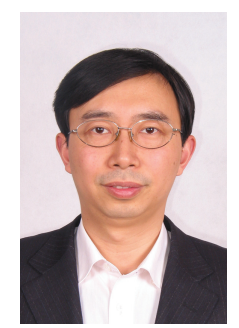

Bin Jiang received the Ph.D. degree in au tomatic control from Northeastern University, Shenyang, China, in 1995. He had been a PostDoctoral Fellow, a Research Fellow and a Visiting Professor in Singapore, France, USA, and Canada, respectively. He is currently a Chair Professor of the Cheung Kong Scholar Program, Ministry of Education, and the Dean of College of Automation Engineering, Nanjing University of Aeronautics and Astronautics, China. His research interests include fault diagnosis and faulttolerant control and their applications in aircraft, satellite and highspeed trains. He is an Associate Editor or Editorial Board Member for a number of journals, such as IEEE TRANSACTION ON CONTROL SYSTEMS TECHNOLOGY, International Journal of Control, Automation and Systems, Nonlinear Analysis, Hybrid Systems, Acta Automatica Sinica, Journal of Astronautics; Control and Decision, and Systems Engineering and Electronics Technologies.

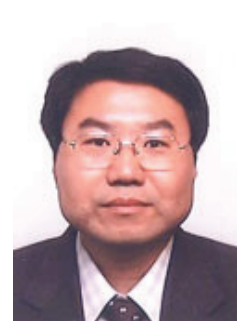

Xing-Gang Yan received the B.Sc. degree from Shaanxi Normal University in 1985, the M.Sc. degree from Qufu Normal University in 1991, and the Ph.D. degree in engineering from Northeastern University, China, in 1997. He was a Lecturer with Qingdao University, China, from 1991 to 1994. He was a Research Fellow/Associate with the University of Hong Kong, China, with Nanyang Technological University, Singapore, and with University of Leicester, U.K. $\mathrm{He}$ is currently a Senior Lecturer with University of Kent, U.K. He is the Editor-in-Chief of International Journal of Engineering Research and Science \& Technology. His research interests include sliding mode control, decentralised control, fault detection and isolation, and control and observation of nonlinear systems and time delay systems with applications.

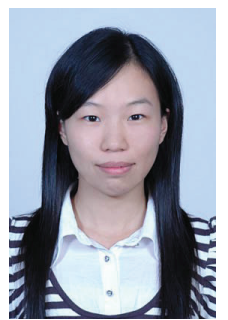

Zehui Mao received the Ph.D. degree in control theory and control engineering from Nanjing University of Aeronautics and Astronautics, Nanjing, China, in 2009. She is currently an Associate Professor with the College of Automation Engineering, Nanjing University of Aeronautics and Astronautics. She is also a Visiting Scholar with the University of Virginia. She was involved in the area of fault diagnosis, with particular interests in nonlinear control systems, sampleddata systems and networked control systems. Her research interests include fault diagnosis and fault-tolerant control of systems with disturbance and incipient faults, and high speed train and spacecraft flight control applications. 